\title{
A topological hierarchy-based approach to layered manufacturing of functionally graded multi-material objects
}

\author{
S. H. Choi ${ }^{*}$ and H. H. Cheung \\ Department of Industrial and Manufacturing Systems Engineering \\ The University of Hong Kong, \\ Pokfulam Road, Hong Kong.
}

\begin{abstract}
This paper presents an approach based on topological hierarchy to representation and subsequent fabrication of functionally graded multi-material (FGM) objects by layered manufacturing. The approach represents an FGM object by material control functions and discretisation of slice contours. Based on the topological hierarchy of slice contours, material control functions are associated with contour families of some representative layers across the X-Y plane and along the Z-plane. The material composition at any location is calculated from the control functions, and the slice contours are discretised into sub-regions of constant material composition. The discretisation resolution can be varied to suit display and fabrication requirements. In comparison with pixel- or voxel-based representation schemes, this approach is computationally efficient, requires little memory, and facilitates fabrication of large and complex objects, which can be assemblies of FGM and discrete materials. The proposed approach has been incorporated with a virtual prototyping system to provide a practical and effective tool for processing FGM objects.
\end{abstract}

Keywords: Topological hierarchy, slice contour, functionally graded, multi-material, layered manufacturing, virtual prototyping

\footnotetext{
*Corresponding author Tel: (852)2859 7054; Fax: (852) 2858 6535, e-mail: shchoi@hku.hk
} 


\section{Introduction}

Multi-material (or heterogeneous) objects may be classified into two major types, namely (i) discrete multi-material (DMM) objects with a collection of distinct materials, and (ii) functionally graded multi-material (FGM) objects with materials that change gradually from one type to another. In comparison with single-material objects, a DMM object can clearly differentiate one part from others, or tissues from blood vessels of a human organ, while an FGM object often performs better in rigorous environments. In particular, suitably graded composition transitions across multi-material interfaces can create an object of very different properties to suit various applications [1,2]. Therefore, it is imperative to develop a practicable manufacturing system to fabricate multi-material objects.

Layered Manufacturing (LM) has been widely explored for fabrication of multimaterial objects. Some experimental multi-material layered manufacturing (MMLM) machines, such as a discrete multiple material selective laser sintering ( ${ }^{2}$ SLS) machine $[3,4]$, a shape deposition manufacturing machine $[5,6]$, a fused deposition of multiple ceramics (FDMC) machine [7], and a 3D inkjet-printing machine [8-10] have been developed. Although these systems seemed suitable for relatively simple objects of a limited variety of materials, they provided a good foundation for further hardware development. The development of MMLM is perhaps more of a software issue than of a hardware problem; it is essential to develop an integrated software system for modelling and fabrication of complex multi-material objects.

In the past decade, some methods for modelling multi-material objects have been developed. Kumar and Dutta $[1,11]$ proposed a mathematical model, called $r_{\mathrm{m}}$-object, by enhancing the theory of r-sets to represent heterogeneous objects. While this model suited DMM objects, it was not quite suitable for FGM objects. Chiu and Tan [12] developed a modified STL file format in which a material tree structure was used to represent a DMM object. The modified STL file, however, became large and slow to process. Jackson [13] proposed a generalized cellular decomposition approach to modelling FGM objects, in which a traditional CAD model was decomposed into a collection of cells, each of which had an analytic function to define the material composition gradient. The topology and composition of the cells were maintained by a cell-tuple data structure. This approach was computationally costly, and it required 
huge memory to represent complex objects. Siu and Tan [14] developed a 'sourcebased' scheme with a 'grading source' to represent FGM objects. A container was used to keep the material composition information, which was controlled by the grading source. Zhou et al. [15] developed a STEP-based approach in which the material variations of an FGM object were assigned by material distribution functions and reference features. Similarly, it could only process objects with simple shapes and composition variations. Kou and Tan [16] developed a heterogeneous feature tree (HFT) for constructive heterogeneous objects. The material variation dependencies among all features, such as points, lines, and faces at different hierarchies, were maintained with an organized structure of a collection of nodes. A recursive material evaluation algorithm based on the HFT was developed to evaluate the material compositions at specific locations. However, the algorithm was computationally intensive and required large memory for handling complex objects.

In addition to the FGM representation schemes above, some researchers have attempted to develop algorithms to process FGM models for fabrication control. A dithering algorithm [9] was developed to convert an FGM model into printable discrete geometry and composition information as a bitmap-like data structure for 3D printing of the FGM object. This algorithm was only suitable for pointwise-based MMLM machines and it needed much memory for complex objects. Siu and Tan [17] outlined a contour sub-division algorithm on each layer arising from slicing a heterogeneous object. However, it could only process relatively simple heterogeneous objects. Shin and Dutta [18] proposed a discretisation-based process planning method for deposition-based fabrication of FGM objects. It converted continuous material variation into stepwise variations. However, this method did not illustrate how to organize the resulting discrete regions with the corresponding constant material compositions to generate efficient toolpaths to control deposition-based MMLM machines. Kou [19] proposed an adaptive sub-faceting method to generate meshbased 2D slices with material composition variation information of an FGM object for visualisation. However, it required huge memory to process complex FGM objects. Kou also decomposed an FGM model into a collection of homogeneous voxels to facilitate fabrication of the FGM object. Again, the voxel-based approach required huge memory for complex objects with fine material gradients. Moreover, it would be 
a time-consuming task to group random voxels with different constant material compositions in each layer for efficient fabrication control.

Overall, the FGM representation schemes and fabrication processing algorithms above tended to be computationally slow and needed large memory, and they were not particularly suitable for complex multi-material objects. This problem indeed warrants research effort to address the main software issue of developing practicable MMLM technology.

\section{The virtual prototyping system for design and fabrication of FGM objects}

This paper therefore proposes a topological hierarchy-based approach to processing functionally graded multi-material (FGM) objects. The proposed approach has been incorporated with a virtual prototyping (VP) system for design, multi-toolpath planning, and subsequent fabrication of FGM objects on MMLM machines. It represents an FGM object with material control functions and discretisation of hierarchical slice contours. Based on the topological hierarchy information of slice contours, material control functions are associated with contour families of some representative layers across the X-Y plane and along the Z-plane. As such, the material composition at any location can be easily calculated from the control functions. For visualisation and subsequent fabrication of FGM objects, the slice contours are discretised into sub-regions of constant material composition. The discretisation resolution, hence the size of sub-regions, can be varied to suit visualisation and fabrication requirements. Figure 1 shows the flow of the proposed approach.

Firstly, a model in STL format, which is normally obtained from a traditional CAD design or digitised images but contains no material information, is sliced; the resulting contours are then sorted to build explicit topological hierarchy information that defines the containment relationship of a group of external and internal contours such that these contours can be simply treated as one or more solid areas. Secondly, the contours are processed for representation of FGM objects, with the following steps: (1) select a number of feature contour families in a representative layer; (2) specify control functions for material variation across layers along the Z-plane in the build direction; (3) specify control functions for material variation from one contour to 
another in the X-Y plane; and (4) discretise the slice contours into sub-regions of constant material composition. Thirdly, the resulting hierarchical contour-based FGM model containing both geometric and material composition information is processed for visualisation, analysis, and fabrication of FGM objects.

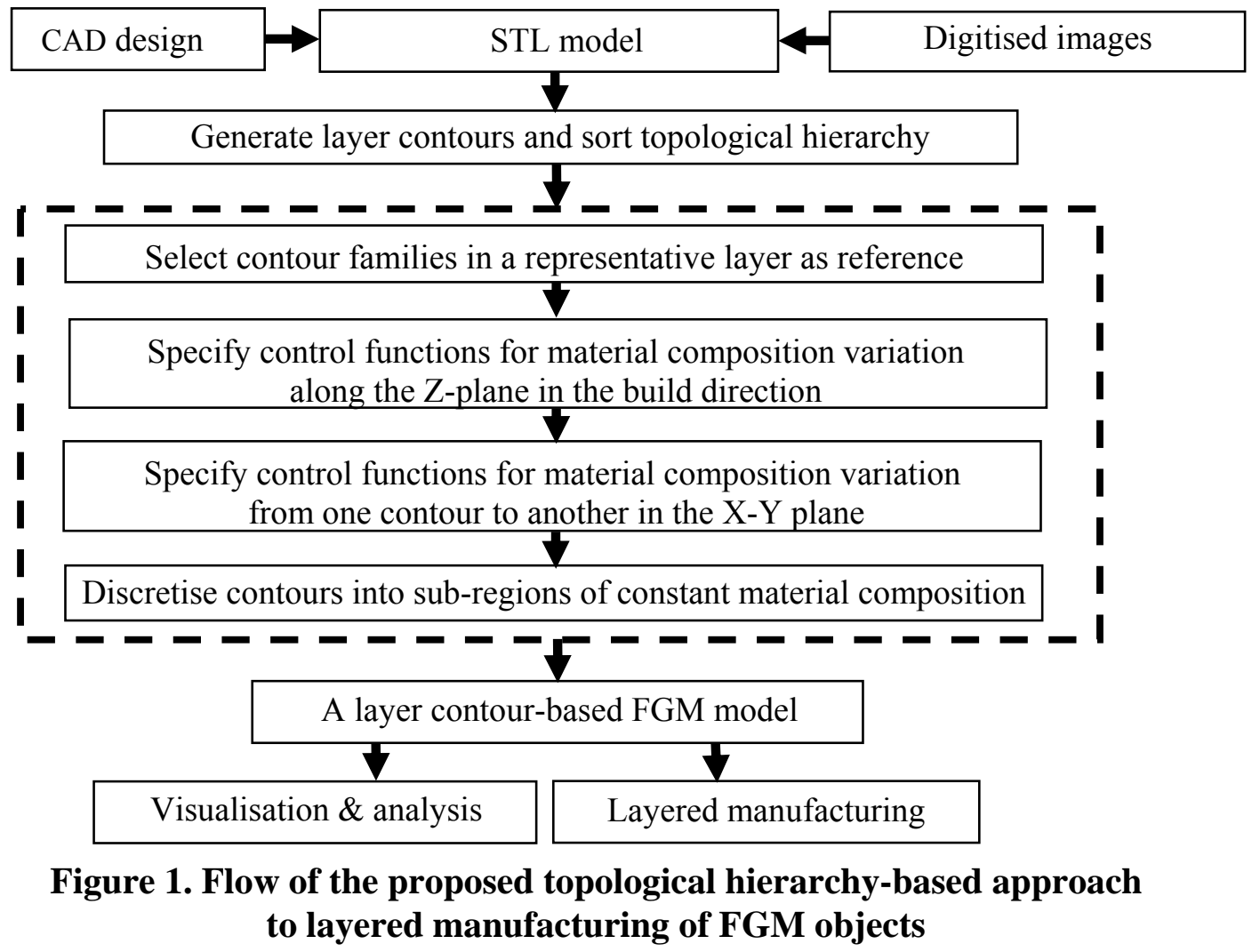

In comparison with pixel- or voxel-based representation schemes, the proposed approach is computationally efficient and requires little memory for processing complex and large objects. This is because pixel- or voxel-based schemes would need to decompose a solid area into a large number of pixels and assign specific material composition information to each pixel, while the proposed approach can simply discretise the solid area into a relatively much smaller number of sub-regions of uniform material. More importantly, the discretisation resolution can be varied to speed up display and facilitate toolpath planning for physical fabrication of both discrete and functionally graded multi-material objects on MMLM machines. In the following sections, a gear assembly is used to illustrate this approach.

\subsection{The topological hierarchy-based approach to processing FMG objects}

The proposed approach firstly slices the STL model of a gear assembly into layer contours. Figure $2 \mathrm{a}$ shows a gear assembly, sliced into twelve layers along the Z- 
plane. Each layer is a homogeneous contour-based model $\left(O_{L}\right)$ which can be represented as a collection of contours $(L)$ :

$$
O_{L}=\left\{\left(L_{i}\right) ; \quad i=1, \ldots, l\right\}
$$

where $L_{i}$ is the $\mathrm{i}^{\text {th }}$ layer, and $l$ is the total number of layers of the object.

A layer $L_{i}$ can be represented as a collection of 2D contours $(C)$ :

$$
L_{i}=\left\{\left(C_{i, j}\right) ; j=1, \ldots, c\right\}
$$

where $C_{i, j}$ is the $\mathrm{j}^{\text {th }}$ contour of the $\mathrm{i}^{\text {th }}$ layer $L_{i} ; c$ is the total number of contours in the $i^{\text {th }}$ layer.

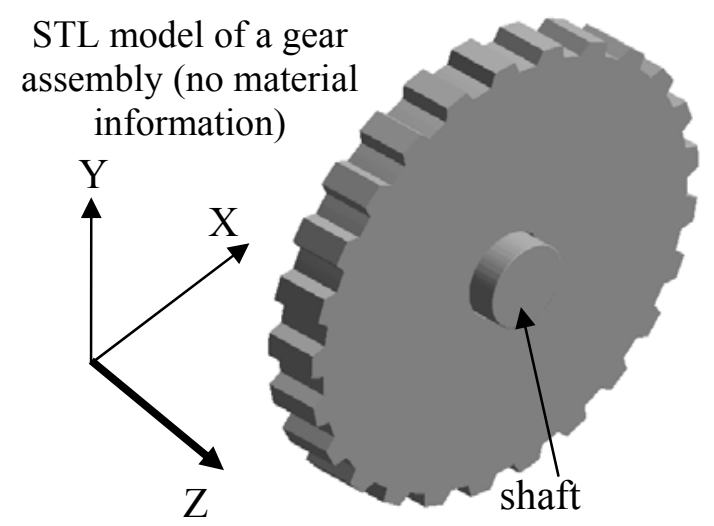

Figure 2a. Slicing a gear assembly to generate a contour-base model

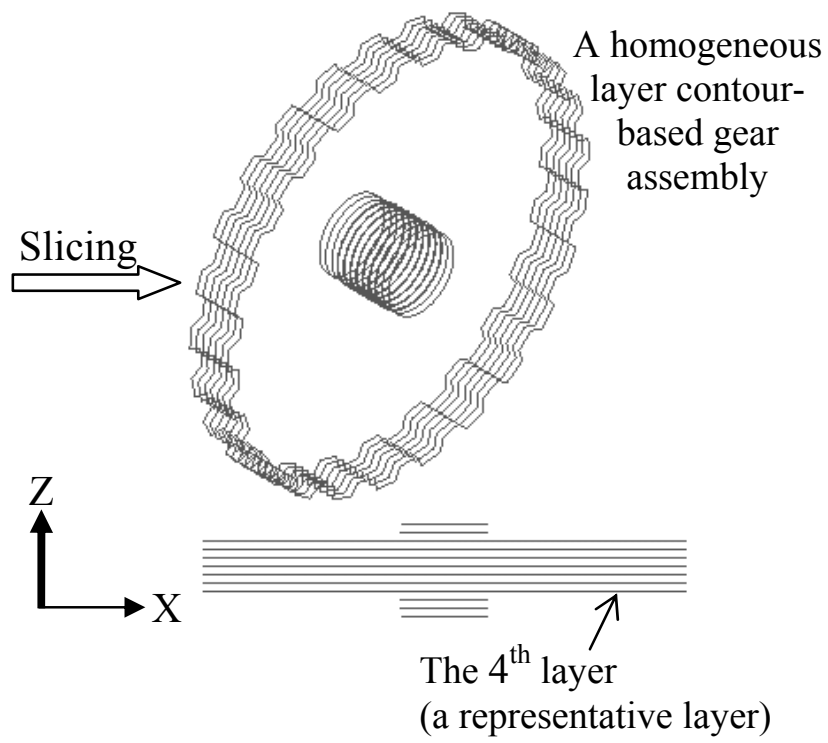

Contour family $P_{1} \rightarrow\left\{f_{1,1}\left(r_{i}\right), f_{1,2}\left(r_{i}\right)\right\}$

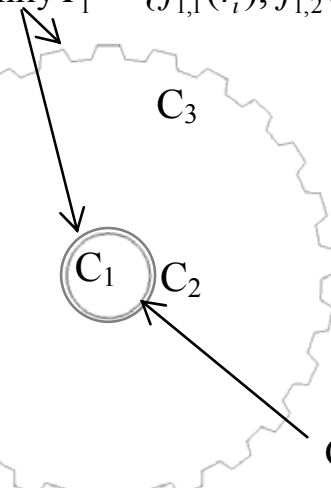

The $4^{\text {th }}$ layer contours of the gear assembly

\begin{tabular}{lc|l}
\hline \multicolumn{2}{r|}{$\begin{array}{r}\text { Parent-and-child list for } \\
\text { contour containment }\end{array}$} & Contour families \\
\hline Level 0 & $\mathrm{C}_{3}$ & 1. $P_{1}\left(\mathrm{C}_{3} \rightarrow \mathrm{C}_{2}\right)$ \\
Level 1 & $\mathrm{C}_{2}$ & 2. $P_{2}\left(\mathrm{C}_{1}\right)$ \\
Level 2 & $\mathrm{C}_{1}$ & \\
\hline
\end{tabular}

Topological hierarchy relationship of contours Contour family $P_{2} \rightarrow\left\{f_{2,1}\left(r_{i}\right)\right\}$

To use a hierarchical contour-based model for representing an FGM object, the material composition and its variations should be incorporated into layer contours accordingly. To do this, the hierarchy relationship of the contours is first built with the 
topological hierarchy-sorting algorithm developed by the authors [21]. Figure $2 b$ shows the fourth layer with three contours, $\mathrm{C}_{1}, \mathrm{C}_{2}$, and $\mathrm{C}_{3}$, sorted into three levels. A contour at Level 0 , such as $C_{3}$, has no parent; $C_{3}$ has two children, $C_{1}$ and $C_{2}$, meaning that $\mathrm{C}_{1}$ and $\mathrm{C}_{2}$ are inside $\mathrm{C}_{3}$. $\mathrm{C}_{2}$ is at Level 1 and has one child, $\mathrm{C}_{1}$; in other words, $\mathrm{C}_{1}$ is inside $C_{2} . C_{1}$ is a Level 2 contour and has no child.

This means a Level 2 contour is of the third generation. This hierarchy establishes a parent-and-child relationship $(P)$ that defines contour containment. Isolating the contours of one generation of parent-and-child relationship, such contours can be regarded as containing 2 contour families, namely family $1\left(\mathrm{C}_{3} \rightarrow \mathrm{C}_{2}\right)$ and family 2 $\left(\mathrm{C}_{1}\right)$, denoted as $P_{1}$ and $P_{2}$, respectively. ( $P$ ) represents a collection of contour families. Each family defines a 2D solid area formed by one or more contours. The parent-and-child relationship of a collection of contour families $(P)$ can be incorporated into Eq. (1), which represents a contour-based model:

$$
O_{L}=\left\{\left(L_{i}, P_{i, k}\right) ; \quad i=1, \ldots, l ; k=1, \ldots, p\right\}
$$

where $p$ is the total number of contour families in the $i^{\text {th }}$ layer.

Based on such topological hierarchy information, primary materials and mathematical control functions can be conveniently associated with the corresponding contour families in a representative layer selected for further design and processing of material composition.

An FGM object $O$ is composed of $\beta$ primary materials. The material composition at a point in the object is a blending of $\beta$ primary materials; it is the sum of the volume fractions of all primary materials, represented by a vector $M$ below:

$$
M=\sum_{b}^{\beta} m_{b}=1
$$

where $m_{b}$ is the volume fraction of the $b^{\text {th }}$ primary material.

To associate a geometry feature with its corresponding material composition, a vector $F$ with $\beta$ mathematical control functions is assigned to the corresponding primary materials to calculate the volume fractions. The vector $F$ can be expressed as:

$$
F=\sum_{b}^{\beta} f_{b}=1 ; \quad b=1, \ldots, \beta
$$

where $f_{b}$ denotes the volume fraction of the primary material $m_{b}$. 
For example, using a vector function $F(x)$, a geometric point $x$ can be mapped to its corresponding material composition vector as:

$$
F(x) \equiv M(x)=\left\{m_{b}(x)\right\}
$$

A multi-material assembly model contains $\alpha$ parts; a set of $\alpha$ material composition vectors $\mathrm{M}^{\prime}$ should be used to represent material composition variations. It is assumed that the material composition of a part is independent and does not affect that of another part. Thus, $\mathrm{M}^{\prime}$ can be represented as:

$$
\mathrm{M}^{\prime}=\left\{M_{a} ; \quad a=1, \ldots, \alpha\right\}
$$

where $M_{a}$ is the material composition vector of the $\mathrm{a}^{\text {th }}$ part; $\alpha$ is the total number of parts in an assembly model.

As a result, a multi-material assembly of FGM and DMM parts can be modelled using the proposed approach by incorporating Eqs. (3) to (7), as follows:

$$
O_{L}=\left\{\left(L_{i}, P_{i, k}, \mathrm{M}_{i}^{\prime}\right) ; \quad i=1, \ldots, l ; \quad k=1, \ldots, p\right\}
$$

\subsubsection{Modelling material variation along the Z-plane}

The gear assembly in Figure 2a contains two parts, a gear and a shaft. We assume that while the shaft is made of a discrete material, the material variation of the gear along the Z-plane is a blending of two primary materials. The detailed effect of the resulting property of blending primary materials is beyond the scope of this paper.

Thus, two material composition vectors, $M_{1}$ and $M_{2}$, are used to represent material variations for the gear and the shaft, respectively. The gear is made of two primary materials, namely $m_{\text {red }}$ and $m_{\text {green }}$, with material variation along the Z-plane. The shaft has one primary material $m_{\text {blue }}$ of constant material composition. To represent this assembly, the fourth layer is selected as a representative layer and its parent-andchild relationship is built in Figure 2b. Based on this relationship, two control functions, $F_{1}\left(g\left(z_{i}\right)\right)=\left\{f_{1,1}\left(g\left(z_{i}\right)\right), f_{1,2}\left(g\left(z_{i}\right)\right)\right\}$ and $F_{2}\left(g\left(z_{i}\right)\right)=\left\{f_{2,1}\left(g\left(z_{i}\right)\right)\right\}$, are assigned to the corresponding contour families, $P_{4,1}$ and $P_{4,2}$, respectively. Here, $g\left(z_{i}\right)$ is a distance ratio $r_{i}$ of the perpendicular distance $d_{i}$ from the start layer (the 
fourth layer) to the $i^{\text {th }}$ layer to the distance $d_{\text {tol }}$ from the start layer to the end layer along the Z-plane; it is expressed as:

$$
g\left(z_{i}\right) \equiv r_{i}=\frac{d_{i}}{d_{t o l}}
$$

where $d_{i}=\left|z_{i}-z_{s}\right|$, and $z_{s}$ and $z_{i}$ are the heights of the start layer and the $\mathrm{i}^{\text {th }}$ layer along the Z-plane, respectively. The distance $d_{\text {tol }}$ from the start layer to the end layer is $\left|z_{e}-z_{s}\right|$, and $z_{e}$ is the height of the end layer.

Hence, a control function expressed as $f\left(r_{i}\right)$ is used to define material variation using various mathematical forms, simple examples of which include:

(i) for linear material variation,

$$
f\left(r_{i}\right)=\mu r_{i}+\lambda
$$

where $\mu$ and $\lambda$ are constants;

(ii) for constant material composition,

$$
f\left(r_{i}\right)=\mu
$$

and (iii) for wave-form material variation,

$$
f\left(r_{i}\right)=\mu \sin \left(\lambda r_{i}\right)
$$

Now for the gear, the two elements of the function vector $F_{1}\left(g\left(z_{i}\right)\right)$ can be expressed as $f_{1,1}\left(r_{i}\right)$ and $f_{1,2}\left(r_{i}\right)$ to calculate the volume fractions of the primary materials, $m_{\text {red }}$ and $m_{\text {green }}$, respectively. Using a simple linear control function in Eq. (10) for the gear's first primary material $f_{1,1}\left(r_{i}\right)=r_{i}$, with $\mu$ and $\lambda$ being one and zero, the control function for the second primary material is given by $f_{1,2}\left(r_{i}\right)=1-f_{1,1}\left(r_{i}\right)$, because the sum of volume fractions of the two primary materials is one.

The property value of a primary material is denoted by colour value $[R, G, B]$. If the property values of two primary materials $m_{\text {red }}$ and $m_{\text {green }}$ are $\left[R_{1}, G_{1}, B_{1}\right]$ and $\left[R_{2}, G_{2}, B_{2}\right]$, respectively, then the property value of the composite material, $[R, G, B]_{i}$, generated by blending these two primary materials at the $i^{\text {th }}$ layer, is expressed as: 


$$
\begin{aligned}
{[R, G, B]_{i} } & =f_{1,1}\left(r_{i}\right)\left[R_{1}, G_{1}, B_{1}\right]+f_{1,2}\left(r_{i}\right)\left[R_{2}, G_{2}, B_{2}\right] \\
& =f_{1,1}\left(r_{i}\right)\left[R_{1}, G_{1}, B_{1}\right]+\left(1-f_{1,1}\left(r_{i}\right)\right)\left[R_{2}, G_{2}, B_{2}\right] \\
& =f_{1,1}\left(r_{i}\right)\left[R_{1}-R_{2}, G_{1}-G_{2}, B_{1}-B_{2}\right]+\left[R_{2}, G_{2}, B_{2}\right]
\end{aligned}
$$

For the shaft, $f_{2,1}\left(r_{i}\right)$ is equal to 1 at any layer height since its material composition is uniform.

Figure 3 shows a multi-material gear with material variation along the Z-plane, and a shaft of a discrete material with uniform composition. The property values of the primary materials $m_{\text {red }}$ and $m_{\text {green }}$ are $[255,0,0]$ and $[0,255,0]$, respectively, and that of the primary material $m_{\text {blue }}$ is $[0,0,255]$. The two primary materials $m_{\text {red }}$ and $m_{\text {green }}$ are blended and the material composition for each layer from the fourth layer to the tenth layer changes linearly along the Z-plane. Using the equations above, the property values of material composition for the corresponding layers of the gear can be found to $[0,255,0]_{4},[43,212,0]_{5},[85,170,0]_{6},[128,127,0]_{7},[170,85,0]_{8},[213,42,0]_{9}$, and $[255,0,0]_{10}$, respectively.

The resulting contours with linear material variation facilitate rendering the gear assembly for better visualisation and analysis, as shown in Figure 3b. In addition, Figure 4 shows the gear assembly with material composition changing non-linearly along the Z-plane. For this assembly, a wave-form composition control function $f_{1,1}\left(r_{i}\right)=\sin \left(r_{i} \pi\right)$ in Eq. (12) is used for the gear's first primary material, with $\mu$ and $\lambda$ being one and $\pi$, respectively. Furthermore, the smoothness of material variation along the Z-plane can be controlled easily by adjusting either the total number of layers or the layer thickness.

\subsubsection{Modelling material variation in the $X-Y$ plane}

To represent material variation from one contour to another in the $\mathrm{X}-\mathrm{Y}$ plane, a 2D area bounded by a contour family is discretised into sub-regions by offsetting the contour family. Subsequently, a control function vector $F$ is associated to define material composition for each sub-region. The details are as follows. 


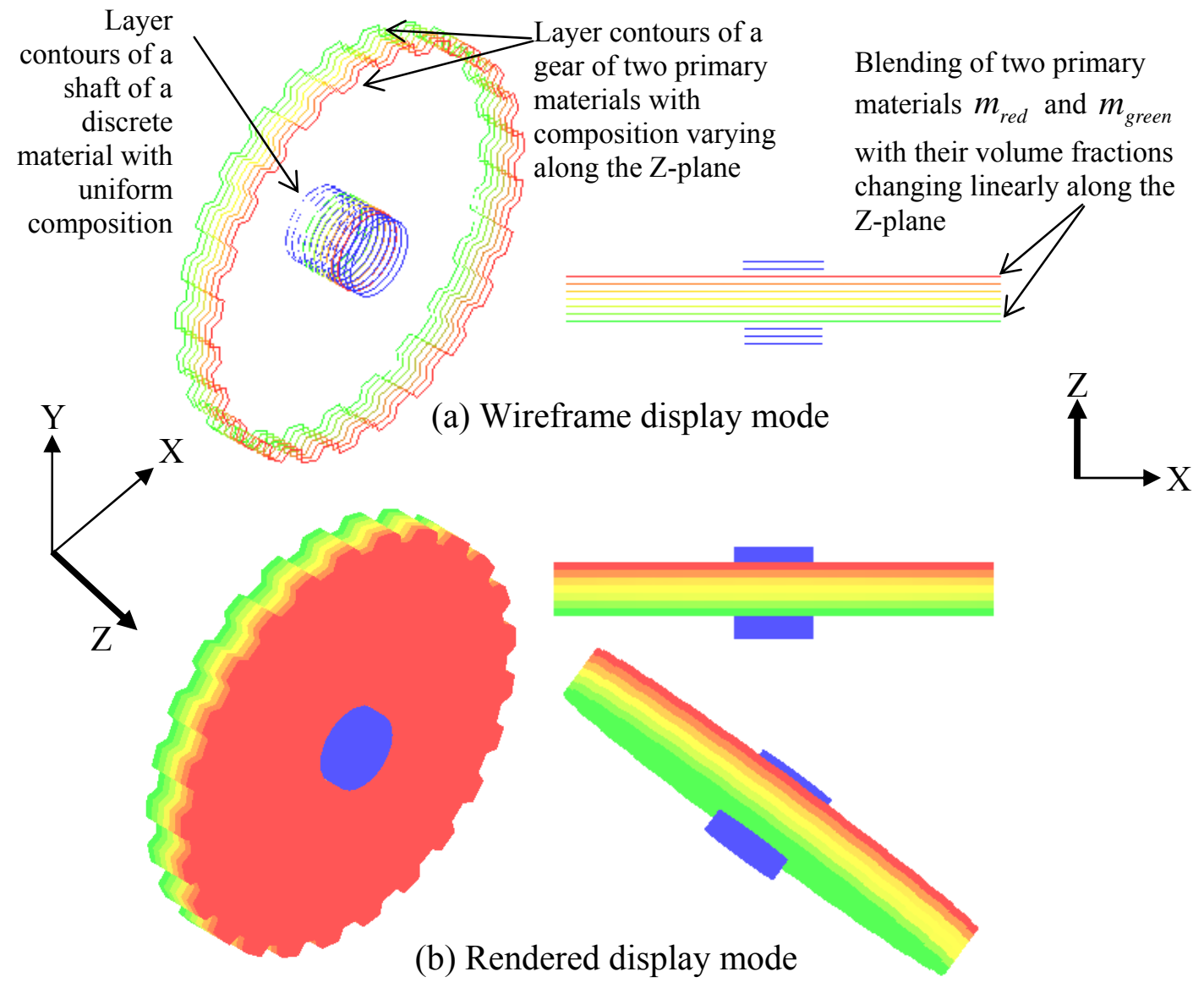

Figure 3. Layer contour-based representation of a multi-material gear assembly

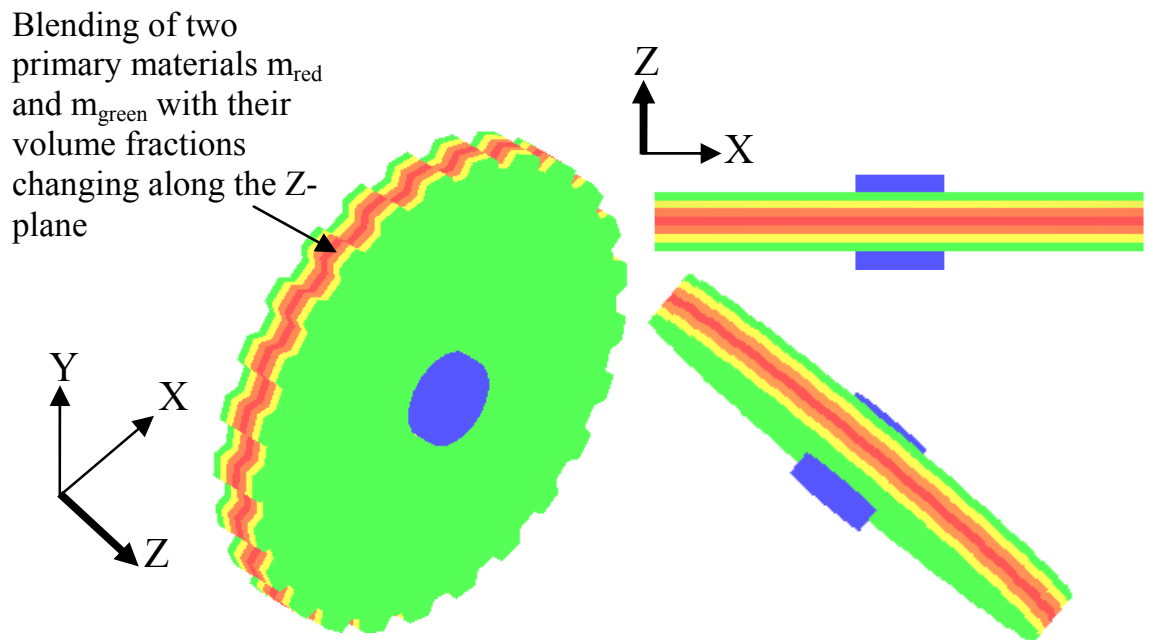

Figure 4. Gear assembly with non-linear material variation along the Z-plane

\subsubsection{Discretisation of slice contours}

Using the topological hierarchy-sorting algorithm [21] mentioned above, slice contours are grouped as a set of contour families with a parent-and-child relationship that defines contour containment. A contour family is a group of external and internal 
contours, which together can be simply treated as a 2D solid area of either constant material property or gradual change of composition by mixing a number of primary materials.

In order to represent material variation, a $2 \mathrm{D}$ solid area is divided into a number of sub-regions, each of which has a constant material composition. Based on the parentand-child relationship, solid areas in each layer can be divided conveniently. Referring to Figure $2 b$, the fourth layer of the gear assembly contains two solid areas; one is bounded by the contour family $P_{1}\left(C_{3} \rightarrow C_{2}\right)$ and the other by the contour family $P_{2}\left(\mathrm{C}_{1}\right)$. Assume that the area bounded by the contour family $P_{1}\left(\mathrm{C}_{3} \rightarrow \mathrm{C}_{2}\right)$ has a gradual change of material composition from an external (a parent) contour $\mathrm{C}_{3}$ to an internal (a child) contour $\mathrm{C}_{2}$, then it is necessary to divide the area into a number of sub-regions. The number of sub-regions $N_{R}$, which determines the discretisation resolution and hence the size of sub-regions, can be chosen to suit visualisation and subsequent fabrication requirements. In general, $N_{R}$ depends primarily on the diameter of a tool or nozzle of an MMLM machine. This means that if a fine material variation is required, a nozzle with small diameter should be used. $N_{R}$ can be denoted as:

$$
N_{R}=\operatorname{int}\left(\frac{L_{S}}{D_{N}}\right),
$$

where $L_{S}$ is the shortest distance of a line $\ell$ from a point of one contour to that of another, i.e. the minimum distance between contours; $D_{N}$ is the diameter of a nozzle.

After determining the number of sub-regions $N_{R}$, a 2D solid area is discretised into $N_{R}$ sub-regions by offsetting the contour family accordingly. For example, Figure 5 shows the area bounded by the contour family $P_{1}\left(\mathrm{C}_{3} \rightarrow \mathrm{C}_{2}\right)$ of the fourth layer in Figure $2 b$ being divided into ten sub-regions, namely $R_{0}, R_{1}, R_{2}, R_{3}, R_{4}, R_{5}, R_{6}, R_{7}, R_{8}$, and $\mathrm{R}_{9}$, respectively, by offsetting the contour $\mathrm{C}_{3}$ towards to the contour $\mathrm{C}_{2}$. These sub-regions are enclosed by the corresponding contours $\left(\mathrm{C}_{3}, \mathrm{C}_{\mathrm{D} 1}\right),\left(\mathrm{C}_{\mathrm{D} 1}, \mathrm{C}_{\mathrm{D} 2}\right),\left(\mathrm{C}_{\mathrm{D} 2}\right.$, $\left.\mathrm{C}_{\mathrm{D} 3}\right),\left(\mathrm{C}_{\mathrm{D} 3}, \mathrm{C}_{\mathrm{D} 4}\right),\left(\mathrm{C}_{\mathrm{D} 4}, \mathrm{C}_{\mathrm{D} 5}\right),\left(\mathrm{C}_{\mathrm{D} 5}, \mathrm{C}_{\mathrm{D} 6}\right),\left(\mathrm{C}_{\mathrm{D} 6}, \mathrm{C}_{\mathrm{D} 7}\right),\left(\mathrm{C}_{\mathrm{D} 7}, \mathrm{C}_{\mathrm{D} 8}\right),\left(\mathrm{C}_{\mathrm{D} 8}, \mathrm{C}_{\mathrm{D} 9}\right)$, and $\left(\mathrm{C}_{\mathrm{D} 9}\right.$, $\mathrm{C}_{2}$ ), respectively. 


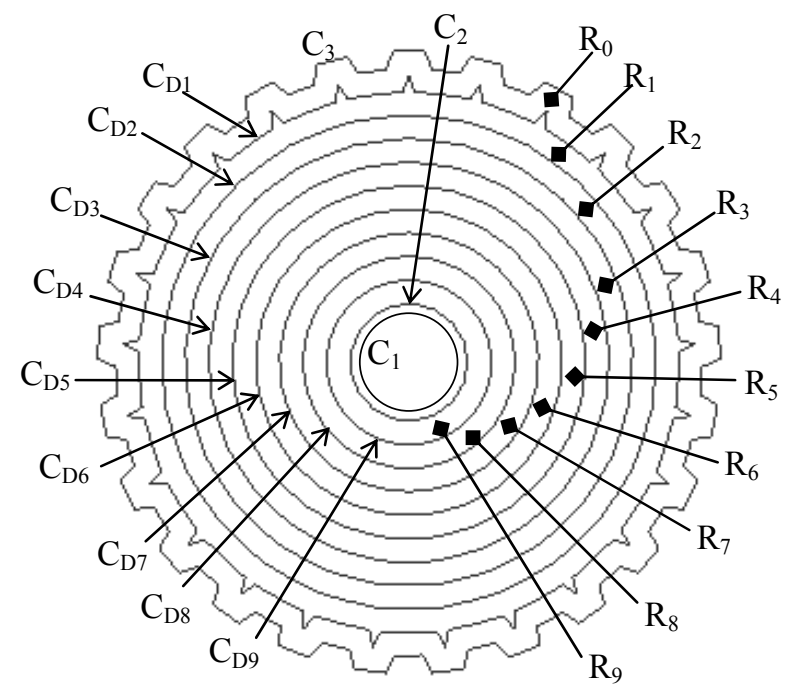

Figure 5. A contour family divided into ten sub-regions

To divide a 2D solid area into sub-regions effectively, it is important to obtain an ordered list of offset-points from the original contours and subsequently form an offset-contour by appropriately connecting the points. Hence, two computing methods are implemented to offset contours.

Assume $C=\left\{v_{i}, 0 \leq i \leq n\right\}$ to be a closed contour composed of $n+1$ points and the start point and the end point are the same, and let $e_{0}=v_{0} v_{1}, e_{1}=v_{1} v_{2}, \ldots$, $e_{i}=v_{i} v_{i+1}, \ldots, e_{n-1}=v_{n-1} v_{n}$ be $\mathrm{n}$ line segments connecting such points and these segments form the contour $C$.

\section{(a) Method 1 for discretisation of slice contours}

In Method 1, an offset-point $v_{i}^{o}$ is calculated from the contour $C$ by:

$$
v_{i}^{o}=v_{i}+d N_{i}
$$

where $N_{i}$ is a unit normal vector of a line segment $e_{i}$ formed by two consecutive points $v_{i}$ and $v_{i+1} ; d$ is an offset-distance equal to $D_{N}$.

Besides, an offset-line segment $e_{i}^{o}$ is calculated by:

$$
e_{i}^{o}=v_{i}^{o}+\omega_{i} D_{i}
$$

where $D_{i}$ is the unit direction vector of $e_{i}$ and $\omega_{i}$ is a parametric value of $0 \leq \omega_{i} \leq 1$. With Eqs. (15) and (16), offset-line segments are easily obtained and then pairwise intersection of individual offset-line segments is used to trim them into an offset- 
contour. Using Method 1, a 2D solid area is divided into ten sub-regions by calculating a number of offset-contours with equal distance between each pair of adjacent contours as shown in Figure 5.

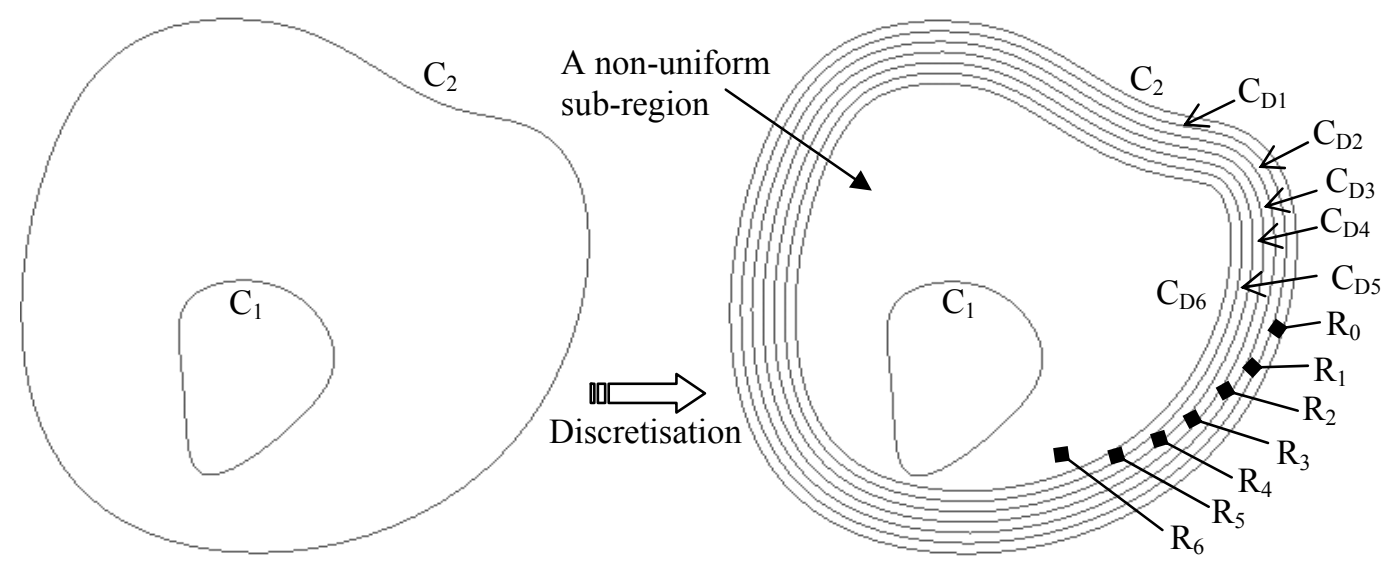

Figure 6. Non-uniform discretisation of an area bounded by two nonconcentric contours by Method 1

Figure 6 shows a 2D solid area bounded by a contour family with two free-form and non-concentric contours $\left(\mathrm{C}_{2} \rightarrow \mathrm{C}_{1}\right)$. With Method 1, the area is partitioned into seven sub-regions, namely $\mathrm{R}_{0}, \mathrm{R}_{1}, \mathrm{R}_{2}, \mathrm{R}_{3}, \mathrm{R}_{4}, \mathrm{R}_{5}$, and $\mathrm{R}_{6}$, respectively; and these sub-regions are bounded by the corresponding contours $\left(\mathrm{C}_{2}, \mathrm{C}_{\mathrm{D} 1}\right),\left(\mathrm{C}_{\mathrm{D} 1}, \mathrm{C}_{\mathrm{D} 2}\right),\left(\mathrm{C}_{\mathrm{D} 2}, \mathrm{C}_{\mathrm{D} 3}\right),\left(\mathrm{C}_{\mathrm{D} 3}\right.$, $\left.\mathrm{C}_{\mathrm{D} 4}\right),\left(\mathrm{C}_{\mathrm{D} 4}, \mathrm{C}_{\mathrm{D} 5}\right),\left(\mathrm{C}_{\mathrm{D} 5}, \mathrm{C}_{\mathrm{D} 6}\right)$, and $\left(\mathrm{C}_{\mathrm{D} 6}, \mathrm{C}_{1}\right)$. Obviously, the discretisation result may not be satisfactory since the resulting sub-regions are not uniform and the sub-region $\mathrm{R}_{6}$ is much larger than other sub-regions.

\section{(b) Method 2 for discretisation of slice contours}

To overcome this weakness, Method 2 is developed to offset contours adaptively, as shown in Figure 7. Here, a set of radial lines $\left\{\ell_{i}^{R}, 0 \leq i \leq n\right\}$ is firstly generated by means of sequentially emitting a ray from the start point of an external contour to centre of an internal contour until the end point of the external contour to facilitate calculation of offset-contours; the total number of radial lines is equal to the total number of points of an external contour. Referring to Figure 7, a ray is emitted from a point $v_{i}$ on an external contour $\mathrm{C}_{2}$ to the centre of an internal contour $\mathrm{C}_{1}$; the ray intersects with the internal contour $\mathrm{C}_{1}$ at a point $v_{i}$. A radial line $\ell_{i}^{r}=v_{i} v_{i}$ is formed by connecting the points $v_{i}$ and $v_{i}$. The resulting radial lines are stored in a data linked list for calculation of offset-contours. If the area is divided into $N_{R}$ sub- 
regions, each radial line should be equally divided into $N_{R}$ segments and the dividing point for each segment on each radial line is an offset-point calculated by:

$$
e_{i j}^{o}=v_{i}+\mathfrak{R}_{j}\left(v_{i}-v_{i}\right)
$$

where $i(0 \leq i \leq n)$ means the $i^{\text {th }}$ radial line and $n+1$ the total number of the radial lines; $j\left(1 \leq j \leq N_{R}\right)$ means the $\mathrm{j}^{\text {th }}$ segment and $N_{R}$ is the total number of segments on a radial line; $\mathfrak{R}_{j}=\frac{j}{N_{R}}$ is a parametric value which is a distance ratio of a distance from $v_{i}$ to the $j^{\text {th }}$ dividing point of the $i^{\text {th }}$ radial line to the length of the $i^{\text {th }}$ radial line.

Now, the area bounded by the contours $\left(\mathrm{C}_{2} \rightarrow \mathrm{C}_{1}\right)$ in Figure 6 is uniformly divided into seven sub-regions as shown in Figure 7 since an offset-contour can be parametrically calculated from a set of radial lines by Eq. (17).
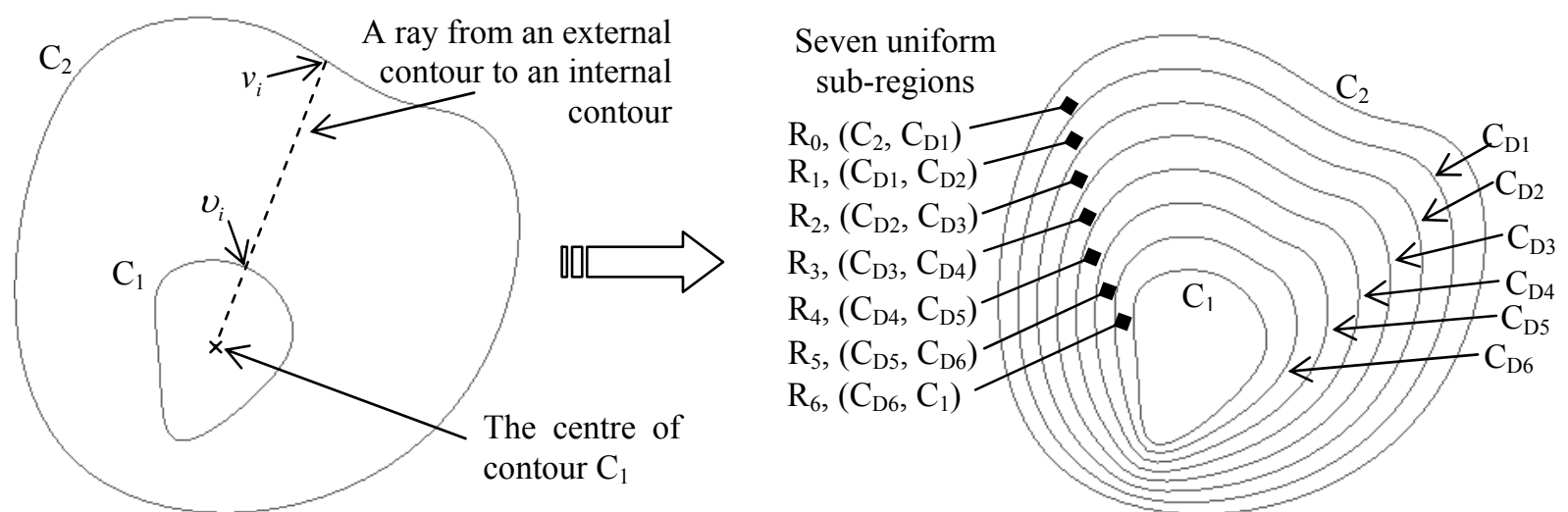

Figure 7. Uniform discretisation of an area into uniform sub-regions by Method 2

\section{(c) Steps for discretisation of slice contours}

While Method 1 suits relatively concentric objects, Method 2 is more appropriate for eccentric ones. The proposed approach incorporates both methods for discretisation of 2D slices to facilitate design and representation of FGM objects. Based on the topological hierarchy information of slice contours, the steps of discretisation for a layer of contours are summarised as:

\section{Define:}

$\mathrm{p}=$ total number of contour families in a layer;

$N_{R}=$ total number of sub-regions in a $2 \mathrm{D}$ solid area bounded by a contour family; 
$\mathrm{i}=$ contour family number in a slice;

$\mathrm{j}=$ sub-region number in a slice;

Steps:

1. Initialise $\xi$ as an empty list of contours;

2. Set the value of $i$ to 1 ;

3. Set the value of $j$ to 1 ;

4. Obtain an offset-contour by either Method 1 or Method 2, based on the user's choice;

5. Insert the contour into the list $\xi$;

6. If $\mathrm{j} \leq N_{R}$, increase the value of $\mathrm{j}$ by 1 , and go to step 4 ; else, increase the value of $i$ by one, and go to step 7;

7. If $\mathrm{i} \leq \mathrm{p}$, go to step 3; else stop.

\subsubsection{Representation of material variation in discretised sub-regions}

To evaluate the property value of material composition of a point in a $2 \mathrm{D}$ solid area bounded by a contour family, it is necessary to incorporate primary material information and material control functions into the contour family. A distance-based approach is adopted for defining material variation in a 2D solid area. It allows the user to choose a set of reference contour features, and to define distance-based control functions for primary materials accordingly. For example, a 2D solid area bounded by the contour family $P_{1}$ in Figure 5 has two contours $\mathrm{C}_{3}$ and $\mathrm{C}_{2}$ with primary materials, $m_{\text {green }}$ and $m_{\text {black }}$, respectively, as two reference features; the values of $m_{\text {green }}$ and $m_{\text {black }}$ are $[R, G, B]_{\text {green }}$ and $[R, G, B]_{\text {black }}$, respectively, as shown in Figure 8. In order to control the variation of two materials $m_{\text {green }}$ and $m_{\text {black }}$ in the area bounded by $\mathrm{C}_{3}$ and $\mathrm{C}_{2}$, a function vector $F_{x y}(r)$ with two control functions $\left\{f_{x y, 1}(r), f_{x y, 2}(r)\right\}$ is assigned to $\mathrm{C}_{3}$ and $\mathrm{C}_{2}$, respectively. Referring to Figure 8 , at a point $\varepsilon, r$ is the distance ratio of the distance, $D_{\varepsilon}$, from the contour $\mathrm{C}_{3}$ to the point $\varepsilon$ along the direction of a radial line from $\mathrm{C}_{3}$ to $\mathrm{C}_{2}$ through the point $\varepsilon$, to the length, $L_{r}$, of the radial line. $r$ can be represented as:

$$
r=\frac{D_{\varepsilon}}{L_{r}}
$$

Now, the control function is $f_{x y, 1}(r)=1-r$, and it becomes $1-\frac{D_{\varepsilon}}{L_{r}}$ with substitution of Eq. (18); and $f_{x y, 2}(r)=1-f_{x y, 1}(r)=1-\left(1-\frac{D_{\varepsilon}}{L_{r}}\right)=\frac{D_{\varepsilon}}{L_{r}}$, since the sum of $f_{x y, 1}(r)$ 
and $f_{x y, 2}(r)$ is equal to one. Thus, the material properties of the red point can be calculated by substituting $f_{x y, 1}(r)$ into Eq. (13) as:

$$
[R, G, B]=f_{x y, 1}(r)\left([R, G, B]_{\text {green }}-[R, G, B]_{\text {black }}\right)+[R, G, B]_{\text {black }}
$$

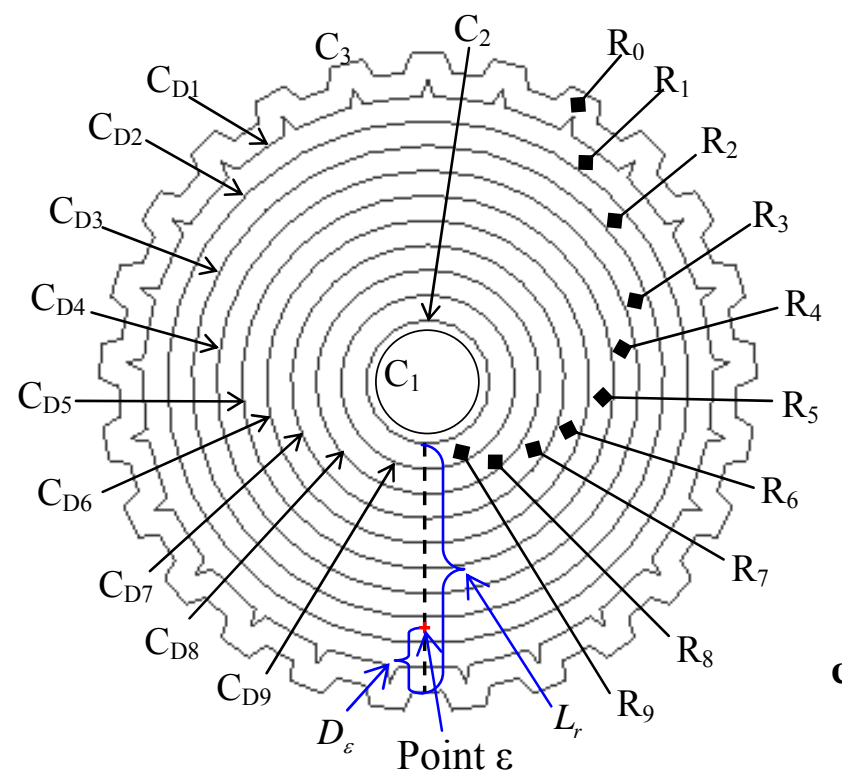

\begin{tabular}{c|c|c}
\hline Contours & $\begin{array}{c}\text { Primary } \\
\text { Materials }\end{array}$ & $\begin{array}{c}\text { Control } \\
\text { functions }\end{array}$ \\
\hline $\mathrm{C}_{3}$ & $m_{\text {green }}$ & $f_{x y, 1}(r)$ \\
\hline $\mathrm{C}_{2}$ & $m_{\text {black }}$ & $f_{x y, 2}(r)$ \\
\hline
\end{tabular}

Figure 8. The distance-based approach for calculation of material composition of a point bounded by two contours

Referring to Figure 8, the area is uniformly discretised into ten sub-regions along the radial direction. Although the material composition changes gradually along a radial direction, it is constant in each sub-region. Therefore, using the Eq. (19), the material properties of the $\mathrm{j}^{\text {th }}$ sub-region can be calculated as:

$$
[R, G, B]_{j}=f_{x y, 1}\left(r_{j}\right)\left([R, G, B]_{\text {green }}-[R, G, B]_{\text {black }}\right)+[R, G, B]_{\text {black }}
$$

where $r_{j}$ is a distance ratio for the $\mathrm{j}^{\text {th }}$ sub-region; using the Eq. (18), it can be calculated as:

$$
r=r_{j}=\frac{D_{j}}{L_{r}}=\frac{j}{N_{R}-1} ; j=0, \ldots, N_{R}-1
$$

where $D_{j}$ is a distance from the $1^{\text {st }}$ sub-region to the $\mathrm{j}^{\text {th }}$ sub-region; $j$ and $N_{R}-1$ can replace $D_{j}$ and $L_{r}$, respectively, since the area is uniformly discretised into $N_{R}$ subregions and each sub-region has a constant material composition. 
As shown in Figure 8, the area is discretised into ten sub-regions, and hence $N_{R}=10$; the values of $m_{\text {green }}$ and $m_{\text {black }}$ are $[0,255,0]_{\text {green }}$ and $[0,0,0]_{\text {black }}$, respectively. Using Eqs. (20) and (21), the material properties of the sub-regions are calculated as follows:

$$
\begin{aligned}
& {[R, G, B]_{0}=\left(1-\frac{0}{10-1}\right)\left([0,255,0]_{\text {green }}-[0,0,0]_{\text {black }}\right)+[0,0,0]=[0,255,0]} \\
& {[R, G, B]_{1}=\left(1-\frac{1}{10-1}\right)\left([0,255,0]_{\text {green }}-[0,0,0]_{\text {black }}\right)+[0,0,0]_{\text {black }}=[0,227,0]} \\
& \ldots \ldots \ldots \ldots . . \\
& {[R, G, B]_{9}=\left(1-\frac{9}{10-1}\right)\left([0,255,0]_{\text {green }}-[0,0,0]_{\text {black }}\right)+[0,0,0]_{\text {black }}=[0,0,0]}
\end{aligned}
$$

The resulting material and geometric contour information are stored layer by layer in a modified CLI file format as shown in Figure 9. In each layer, sets of contours for sub-regions in a 2D solid area bounded by each contour family are stored. In each contour family, the contours are arranged into a sequence from the outermost contour to the innermost contour; a contour identifying number is assigned to each contour (polyline) to identify its sequence; ' 1 ' represents the outermost (parent) contour, ' 0 ' represents the innermost (child) contour; the numbers ' $2,3, \ldots, \mathrm{N}$ ' present their corresponding offset-contours which are arranged from the outermost contour to the innermost contour, respectively; $\mathrm{N}$ is the total number of the offsetting contours. In each polyline (contour), the total number of points is represented by an integer; the value of material properties is represented by a colour value in $[R, G, B]$; and each geometric point is represented by a pair of $(\mathrm{X}, \mathrm{Y})$ coordinates. Using the geometric and material information stored in a modified CLI file, a hierarchical contour-based FGM model can be conveniently processed for visualisation, and inspection of internal material variation of each layer, multi-toolpath planning, and simulation of MMLM processes.

For example, Figure 10 shows a linear variation of material composition in the area bounded by a contour family $\left(\mathrm{C}_{3} \rightarrow \mathrm{C}_{2}\right)$ in Figure 8 . The material composition changes from one sub-region to another. In each sub-region, it has a specific geometric contour and $[R, G, B]$ material information. 


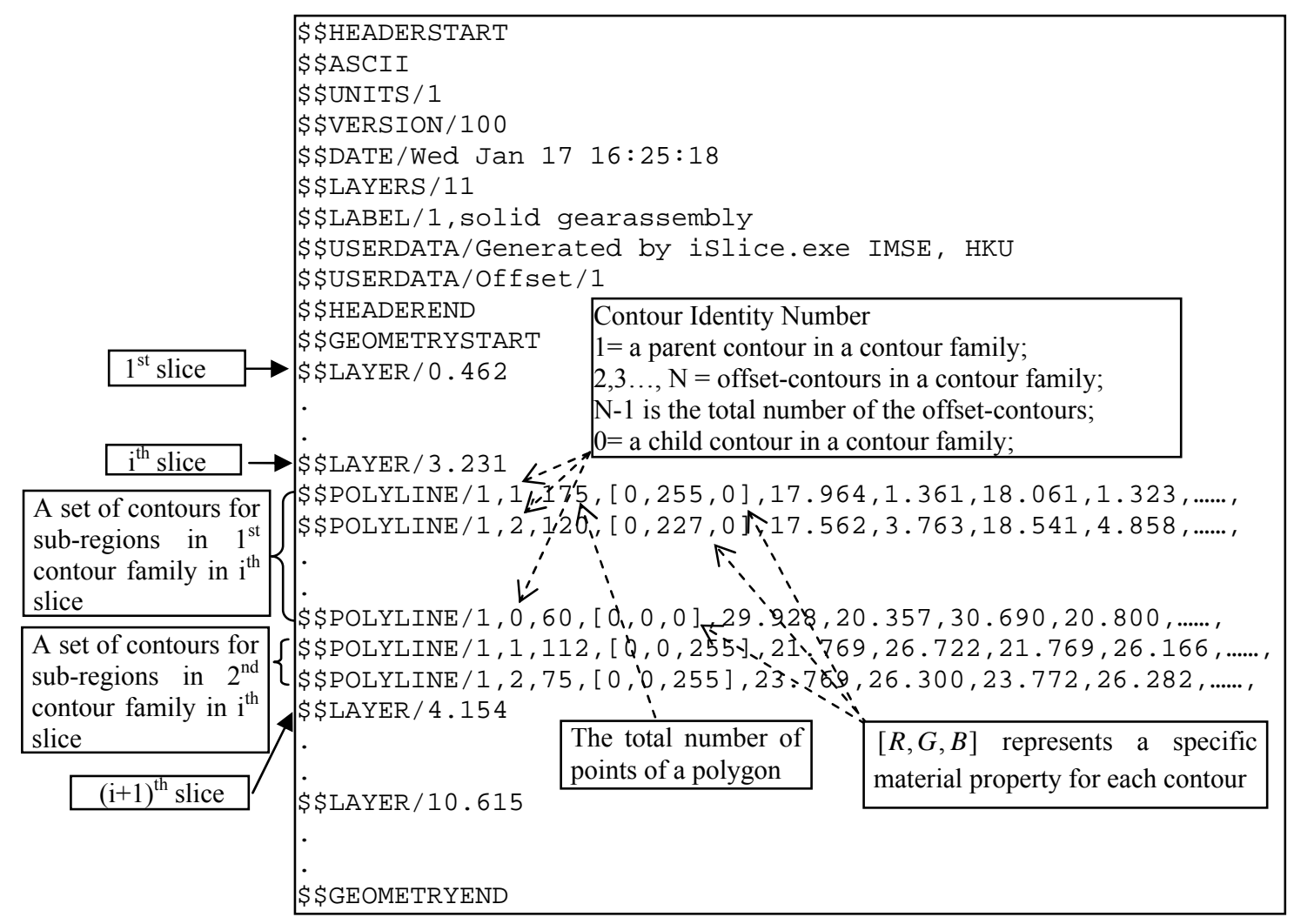

Fig. 9. A modified CLI file format for contour-based FGM models

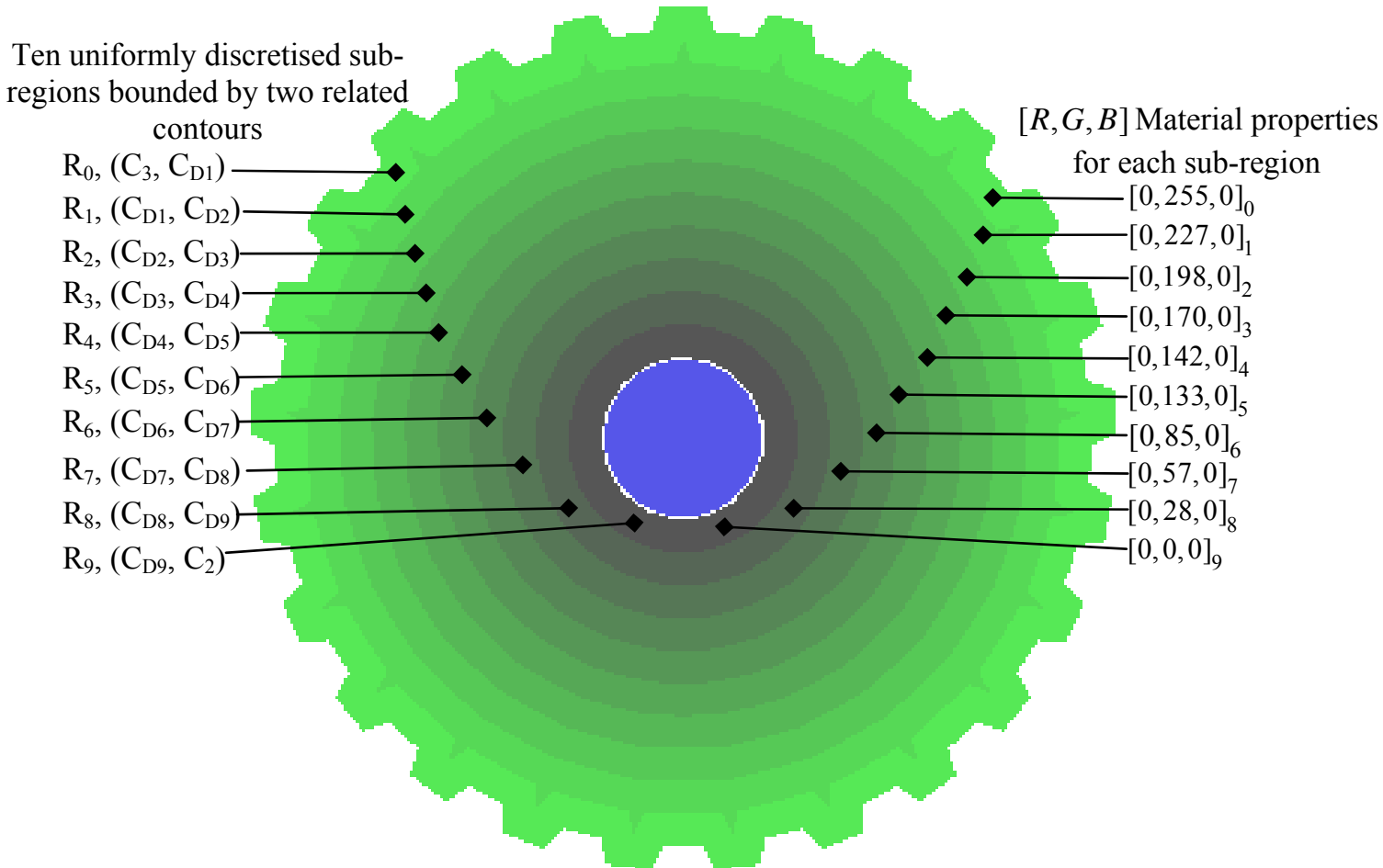

Figure 10. Material variation from one sub-region to another 
Figure 11 shows the gear with material composition varying both along the Z-plane and across the X-Y plane; the shaft is of a discrete material with uniform composition. This figure also highlights the material variation layer by layer across the X-Y plane. Therefore, using the proposed approach, a product of both discrete and functionally graded materials can be conveniently represented for visualisation, analysis, and subsequent fabrication.

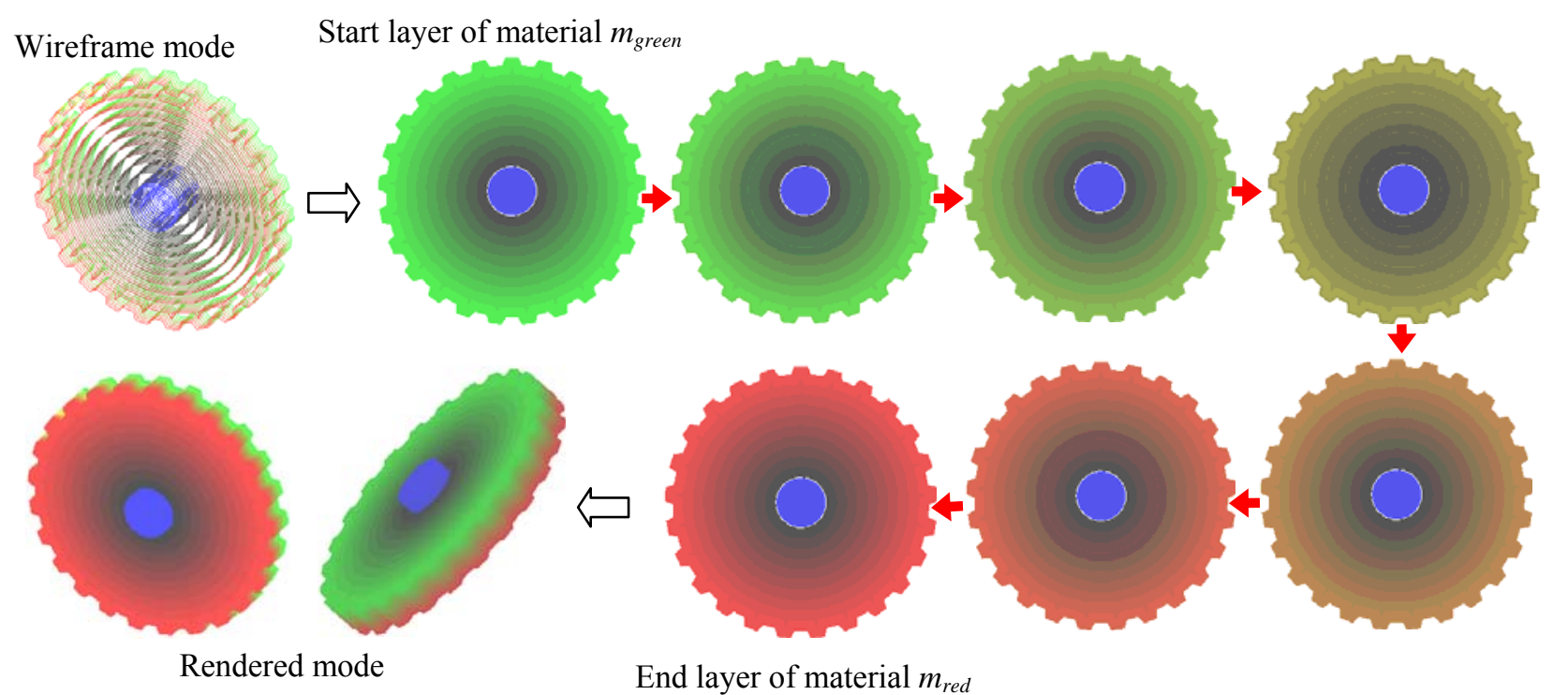

Figure 11. Gear assembly with gradual material variations along the Z-plane and across the $\mathrm{X}-\mathrm{Y}$ plane

In addition, Figure 12 shows a relatively complex slice with a contour family $\left(\mathrm{C}_{1} \rightarrow \mathrm{C}_{2} \rightarrow \mathrm{C}_{3} \rightarrow \mathrm{C}_{4}\right)$ in which the parent (external) contour $\mathrm{C}_{1}$ of a primary material, namely $\mathrm{m}_{1}$ with a property value $[0,0,255]$ and three child (internal) contours $\left(\mathrm{C}_{2}, \mathrm{C}_{3}\right.$, and $\mathrm{C}_{4}$ ) of the same primary material, namely $\mathrm{m}_{2}$ with a property value $[255,0,0]$. In applying Method 2 for uniform discretisation of such a slice with multiple internal contours, a set of radial lines through each internal contour is first generated. Subsequently, the radial lines at regions of adjacent contours are processed to remove any closed loops and sharp turns in the resulting offset-contour. Using this approach, the slice is first divided into ten sub-regions and the material composition of each subregion can then be calculated by Eqs. (20) and (21). A rendered FGM slice is subsequently generated as shown in Figure 12.

However, if the three child contours $\left(\mathrm{C}_{2}, \mathrm{C}_{3}\right.$, and $\left.\mathrm{C}_{4}\right)$ of the slice in Figure 12 have different material properties, namely $\mathrm{m}_{2}, \mathrm{~m}_{3}$, and $\mathrm{m}_{4}$, with property values of $[255,0,0],[0,255,0]$, and $[100,100,0]$, respectively, the material composition changes 
along both the radial and the circumferential directions in the X-Y plane. To present the circumferential material variation, each sub-region is further divided into a set of cells along the circumferential direction, as shown in Figure 13. Each cell has a constant material composition. Indeed, if the cells are very small, they can be regarded as "non-uniform pixels", but an advantage is that the size of the cells can be varied easily to suit display and fabrication requirements.

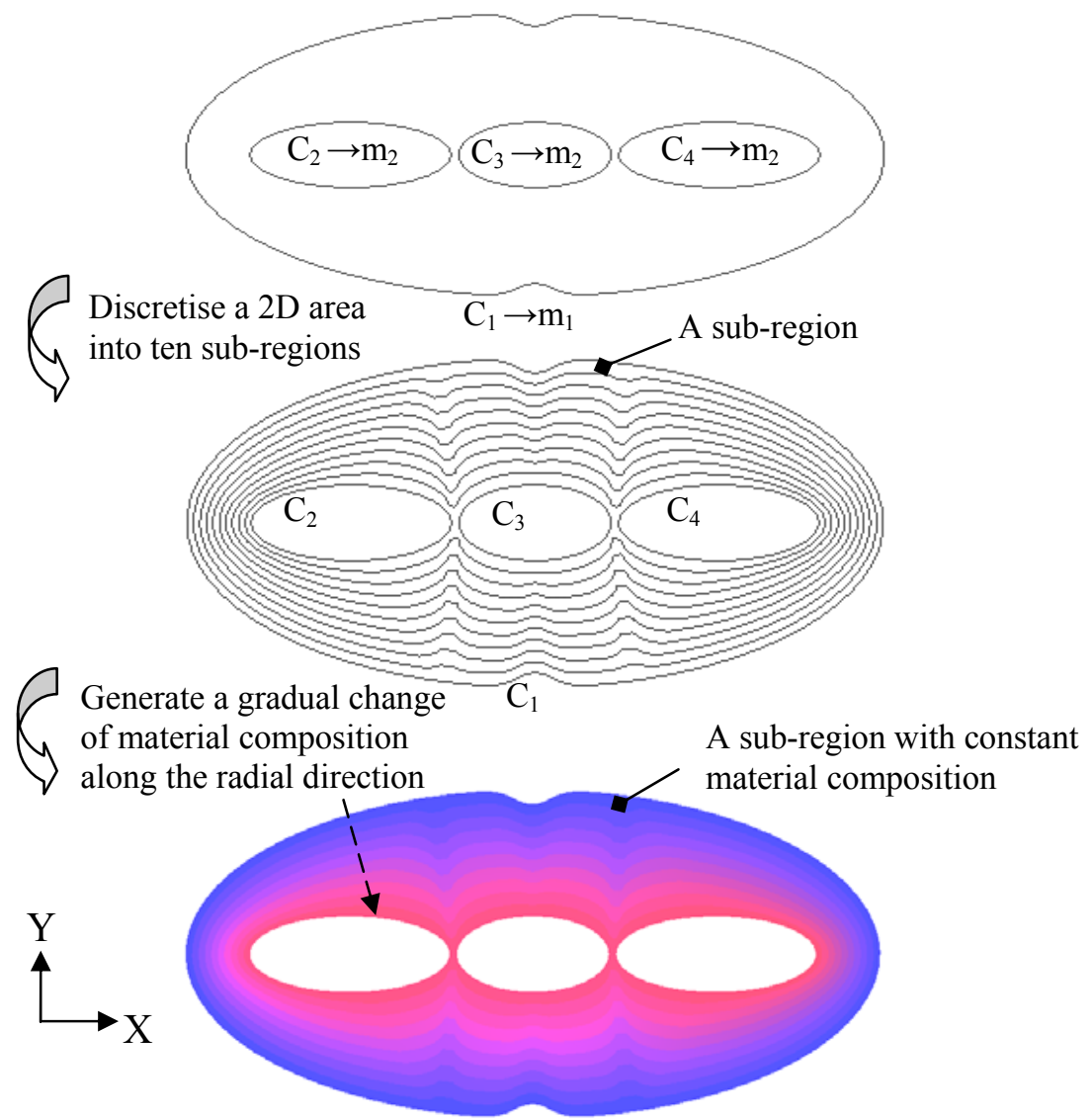

Figure 12. Gradual material variation of a complex slice with four contours

In order to calculate the property value of material composition of each cell, its centre is located as a reference point, as shown Figure 14. Using the inverse distance weighting method $[2,22]$, the property value $M(\varepsilon)$ of material composition of a point $\varepsilon$ is formulated as:

$$
M(\varepsilon)=\sum_{i=1}^{\beta} m_{i}\left(C_{i}\right) \cdot \omega_{i}(\varepsilon)
$$

where $\beta$ is the total number of contours in a contour family; $m_{i}\left(C_{i}\right)$ is the property value of the $\mathrm{i}^{\text {th }}$ primary material for the contour $C_{i} ; \omega_{i}(\varepsilon)$ is the volume fraction of the $\mathrm{i}^{\text {th }}$ primary material at a point $\varepsilon$, and can be calculated as: 


$$
\omega_{i}(\varepsilon)=\frac{\prod_{k=1, k \neq i}^{\beta} d_{k}(\varepsilon)}{\sum_{j=1}^{\beta} \prod_{k=1, k \neq j}^{\beta} d_{k}(\varepsilon)}
$$

where $d_{k}(\varepsilon)$ is the shortest distance from the $\mathrm{k}^{\text {th }}$ contour $C_{k}$ to a point $\varepsilon$, and $\mathrm{k}=1$, $2, \ldots, \beta ; \quad \beta$ is the total number of contours; and

$$
\sum_{i=1}^{\beta} \omega_{i}(\varepsilon)=1
$$

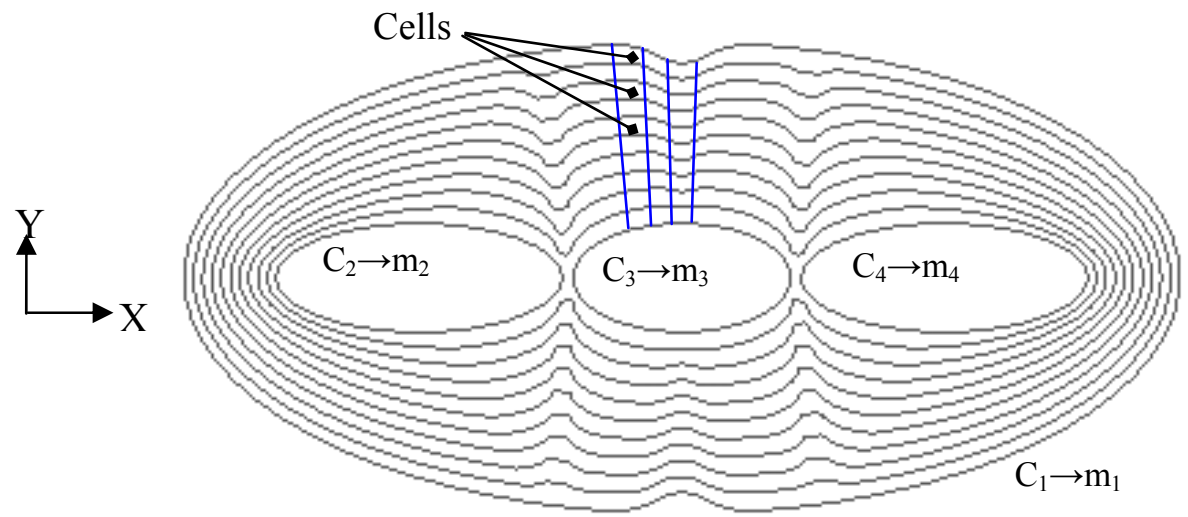

Figure 13. Dividing sub-regions into cells in circumferential direction

Referring to Figure 14, $\beta$ is now equal to 4 and using Eq. (23), the weight of each material at point $\varepsilon$ is calculated accordingly. For example, for $\mathrm{m}_{1}, \omega_{1}(\varepsilon)=\frac{d_{2} d_{3} d_{4}}{d_{2} d_{3} d_{4}+d_{1} d_{3} d_{4}+d_{1} d_{2} d_{4}+d_{1} d_{2} d_{3}}$, and for $\mathrm{m}_{4}, \omega_{4}(\varepsilon)=\frac{d_{1} d_{2} d_{3}}{d_{2} d_{3} d_{4}+d_{1} d_{3} d_{4}+d_{1} d_{2} d_{4}+d_{1} d_{2} d_{3}}$; and $\omega_{1}(\varepsilon)+\omega_{2}(\varepsilon)+\omega_{3}(\varepsilon)+\omega_{4}(\varepsilon)=1$.

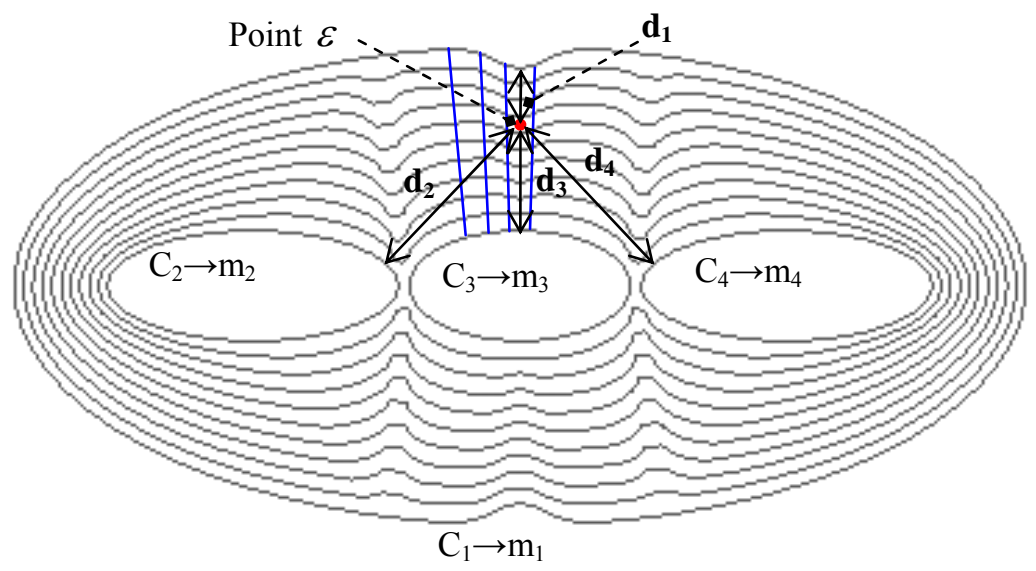

Figure 14. Inverse distance weighting for property value of a point $\varepsilon$ 
Incorporating the above strategy, radial and circumferential material variations can be represented accordingly. As shown in Figure 15, the outermost and the three innermost sub-regions are dominated by their corresponding primary materials, while other sub-regions have gradual changes of material composition along both radial and circumferential directions. Figure 15 also shows that a higher discretisation resolution results in finer material variation. Therefore, the proposed approach can represent FGM objects with detailed material variations in three dimensions, i.e., in radial and circumferential directions and along the Z-plane.

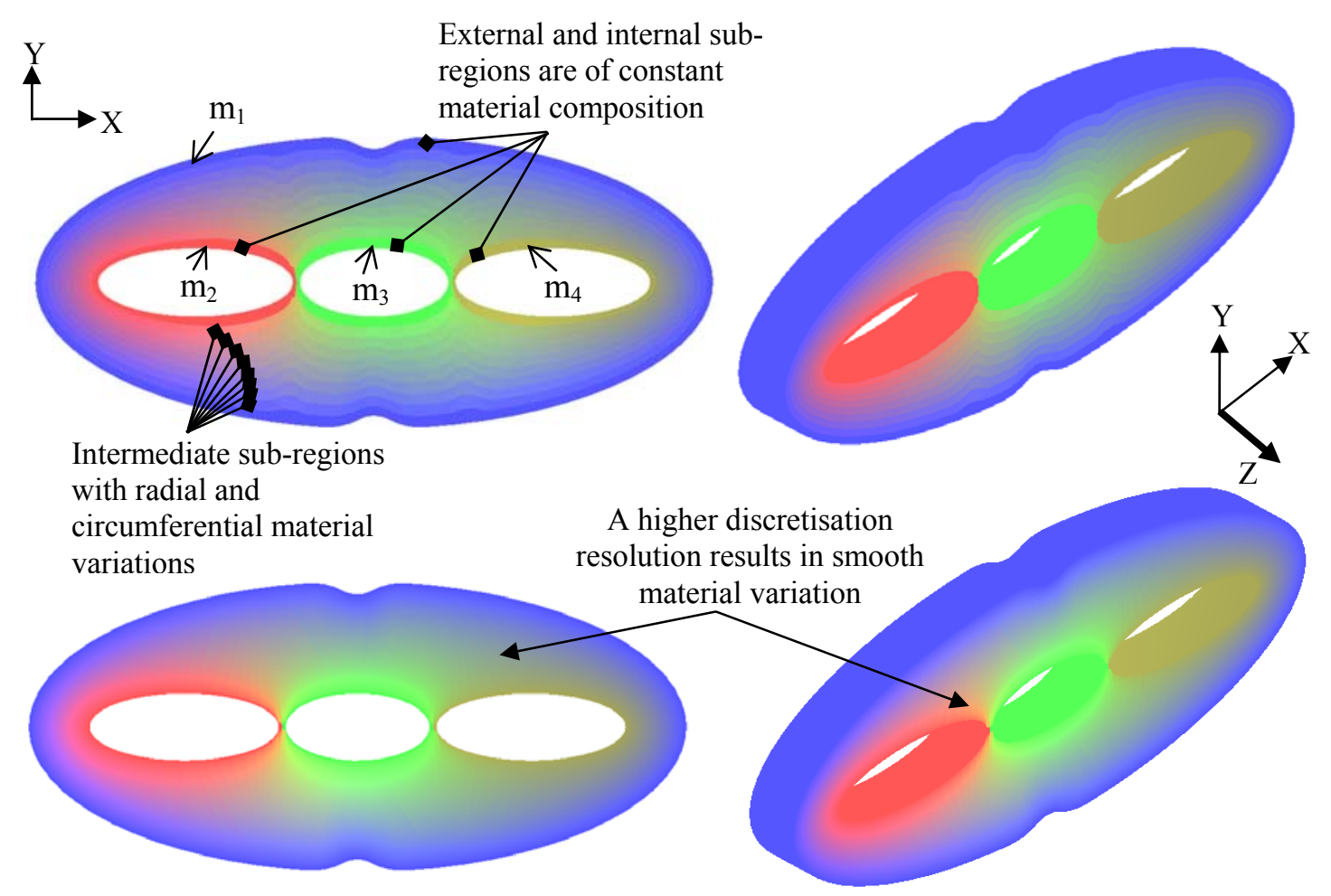

Figure 15. Material variation along both radial and circumferential directions

Subsequently, the FGM object can be processed by the toolpath planning algorithm $[23,24]$ to generate multi-toolpaths for fabrication. Indeed, the discretisation resolution can be conveniently adjusted to control the smoothness of material variation in order to suit fabrication requirements or available resolution of MMLM machines.

\subsection{Fabrication of FGM objects}

The proposed approach has been integrated with a virtual reality (VR) simulation module to form a functionally graded multi-material virtual prototyping (FGMVP) system for design, visualisation, multi-toolpath planning, and digital fabrication of 
FGM objects. Through simulations on the FGMVP system, design assessment and modification of complex FGM products can be iterated without worrying about the manufacturing and material costs of physical prototyping [23-26]. Moreover, the simulation result would facilitate subsequent physical fabrication of the products on MMLM machines.

\section{A Case study}

\subsection{An FGM turbine blade}

Figure 16 shows a turbine blade commonly used in aircraft engines and power generators, in which extreme stresses are often induced by severe thermo-mechanical loading. To alleviate this problem, functionally graded materials have been explored to produce blades with cross-sections of materials changing gradually from ceramics on the high temperature side to metal on the low side. This design enhances heatresistance and anti-oxidation on the high temperature side, and mechanical toughness and strength on the low temperature side, as well as effective thermal stress relaxation throughout the turbine blade [27]. For this purpose, layered manufacturing has been recognised as a high potential method for direct fabrication of FGM objects. Indeed, Das et al. [28] has explored fabrication of super-alloy cermet abrasive turbine blade tips by layered manufacturing process. However, there is no effective way for design and fabrication process planning of complex FGM objects. It would be interesting to explore possible applications of the FGMVP system for such purposes. The turbine blade in Figure 16 is thus used to demonstrate how it can be processed with the FGMVP system.

An STL model of the turbine blade is first sliced into a set of homogeneous layer contours, and explicit topological hierarchy information is then built for each layer. Subsequently, a layer of contours is selected as a feature layer to assign primary materials and material control functions for effective generation of gradual changes of material compositions of the blade. Figure 17 a shows the contours of a selected layer are grouped as a contour family $P_{1}\left(\mathrm{C}_{1} \rightarrow \mathrm{C}_{2}\right)$ and two primary materials, namely ceramics $\left(m_{\text {yellow }}\right)$ and metal $\left(m_{\text {blue }}\right)$, are assigned to contours $\mathrm{C}_{1}$ and $\mathrm{C}_{2}$, respectively. 
A vector $F_{1}\left(r_{j}\right)$ with two control functions $f_{1,1}\left(r_{j}\right)$ and $f_{1,2}\left(r_{j}\right)$ are assigned to control volume fractions of $m_{\text {yellow }}$ and $m_{\text {blue }}$, respectively, and the vector is expressed as:

$$
F_{1}\left(r_{j}\right)= \begin{cases}f_{1,1}\left(r_{j}\right)=1-r_{j}, & 0 \leq r_{j} \leq 1 \\ f_{1,2}\left(r_{j}\right)=r_{j}, & 0 \leq r_{j} \leq 1\end{cases}
$$

where $j$ is the number of sub-regions in a 2D solid area; $r_{j}$ is a distance ratio of the $j^{\text {th }}$ sub-region and can be calculated by Eq. (21).

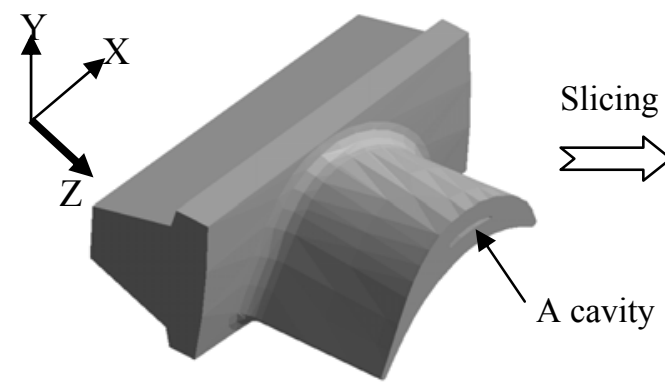

A STL model

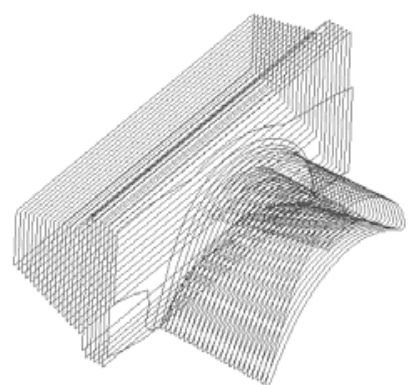

A contour-based model

Figure 16. A turbine blade

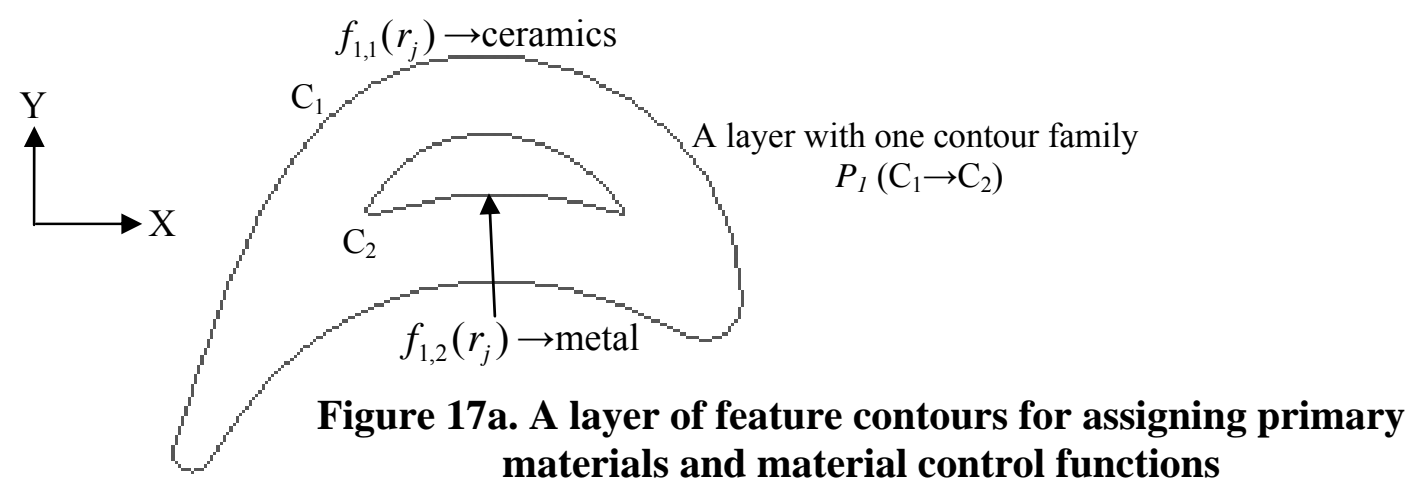
materials and material control functions

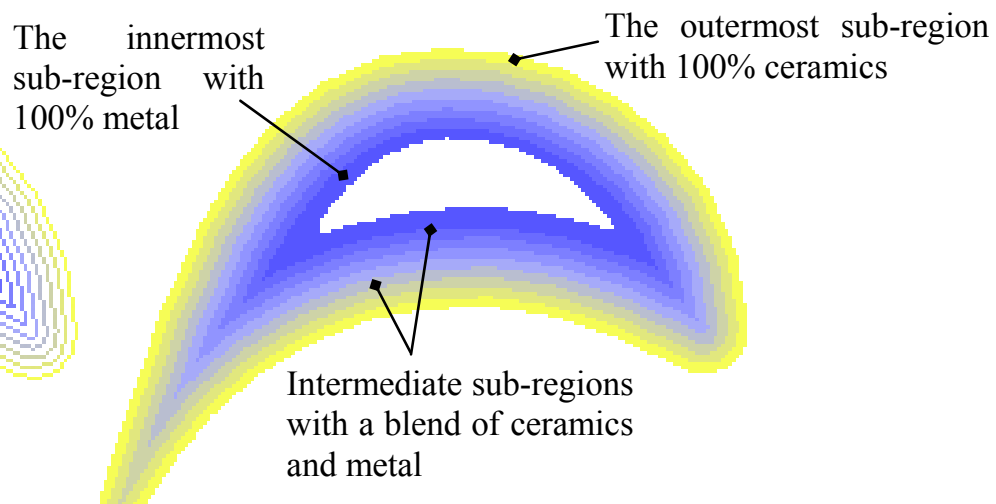

Sub-regions bounded by a set of contours changing from ceramics to metal

Figure 17b. A layer with gradual material variation in the $\mathrm{X}-\mathrm{Y}$ plane 
Subsequently, a $2 \mathrm{D}$ solid area bounded by the contour family $P_{1}\left(\mathrm{C}_{1} \rightarrow \mathrm{C}_{2}\right)$ in the $\mathrm{X}-\mathrm{Y}$ plane is divided into sub-regions using a predefined resolution. At the same time, substituting Eq. (25) into Eq. (20), the property value of material composition of ceramics and metal for each sub-region can be calculated by Eq. (20). The resulting sub-regions bounded by a set of contours with gradual material variation in the X-Y plane is displayed in both wireframe and rendered modes, as in Figure 17b. The material of the outermost region is $100 \%$ ceramics and that of the innermost region is $100 \%$ metal; the intermediate sub-regions exhibit gradual variation from ceramics and metal. This gives the turbine blade desirable heat-resistance and anti-oxidation properties at the external surface and good mechanical toughness and strength at the internal surface; the thermal stresses can also be significantly reduced due to a gradual change of material composition in the intermediate sub-regions. The resulting layer contours with material information stored in the modified CLI file are used for visualisation and digital fabrication of an FGM turbine blade. Figure 18 shows a 3D hierarchical contour-based FGM turbine blade model with a gradual change of material composition in the $\mathrm{X}-\mathrm{Y}$ plane in both wireframe and rendered modes.

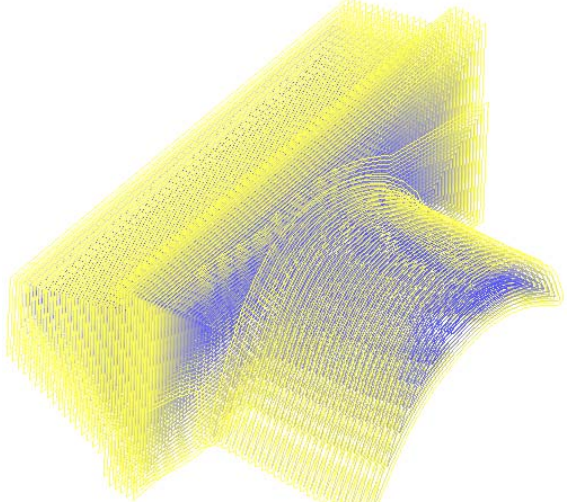

A wireframe display mode

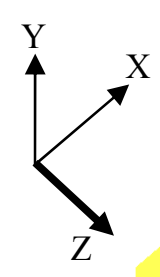

A rendered display mode

Figure 18. A 3D contour-based FGM turbine blade

To represent further material variation along the Z-plane, it is assumed that there is a linear change of material property from relatively ductile to relatively hard, represented by $m_{\text {red }}$ on the first layer to $m_{\text {green }}$ on the last layer along the Z-plane. Similarly, to represent material variation in the $\mathrm{X}-\mathrm{Y}$ plane, two control functions $f_{1,1}\left(r_{j}\right)$ and $f_{1,2}\left(r_{j}\right)$ in Eq. (25) are assigned to control linear changes of volume 
fractions of $m_{\text {red }}$ and $m_{\text {green }}$ along the Z-plane. In this case, $r_{j}$ is replaced by $r_{i}$, which is the ratio of a distance from the first layer to the $\mathrm{i}^{\text {th }}$ layer to the height from the start layer to the end layer along the Z-plane, and can be calculated by Eq. (9); $i$ is the number of layers. Now, by substituting two control functions into Eq. (13), the material composition of each layer can be calculated accordingly. The resulting FGM turbine blade now exhibits 3D material variations along the Z-plane and in the X-Y plane, as in Figure 19. Figure 20 shows the process of digital fabrication of a hierarchical contour-based FGM turbine prototype.

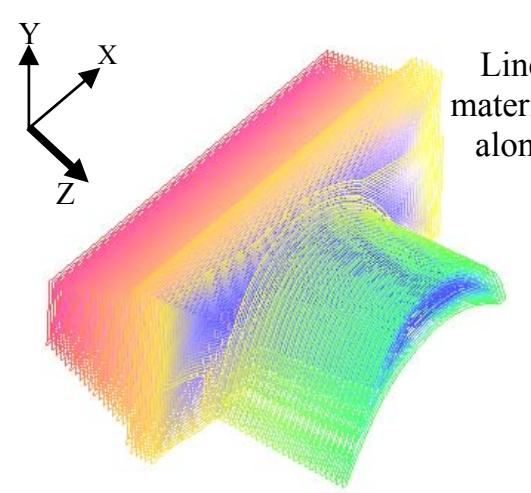

Wireframe mode

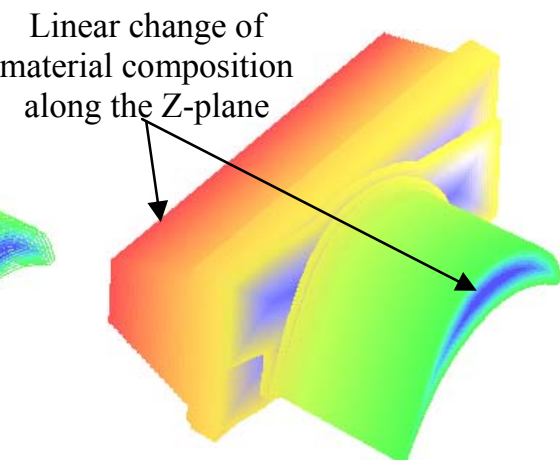

Rendered mode

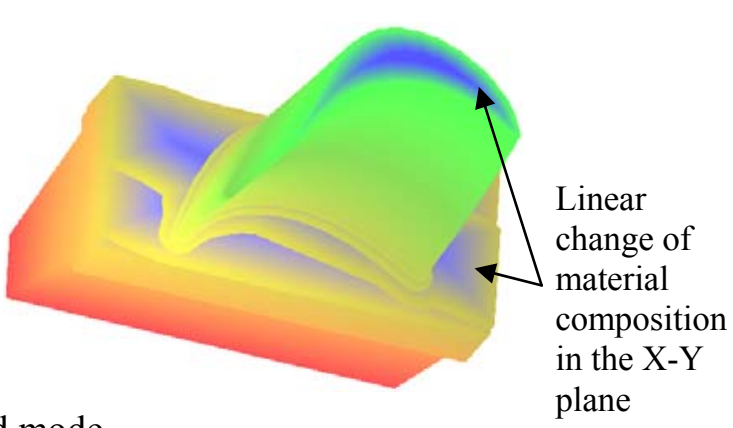

plane

Figure 19. A contour-based FGM turbine blade with 3D variations of material composition along the Z-plane and in $\mathrm{X}$-Y plane

Indeed, the FGMVP system can process objects with more complex shapes and material composition, such as the turbine blade with two cavities for better air cooling, as shown in Figure 21a. Again, a representative layer with a contour family containing three contours $\left(\mathrm{C}_{1} \rightarrow \mathrm{C}_{2} \rightarrow \mathrm{C}_{3}\right)$, in Figure $21 \mathrm{~b}$, is chosen to be assigned three primary materials, $m_{1}, m_{2}$, and $m_{3}$ to the corresponding contours $C_{1}, C_{2}$, and $C_{3}$, respectively. Using Eqs. (22) and (23), the property value of material composition at a point $\varepsilon M(\varepsilon)$ in an area bounded by contours $\mathrm{C}_{1}, \mathrm{C}_{2}$, and $\mathrm{C}_{3}$ can be calculated as follows. By Eq. (22),

$$
M(\varepsilon)=\sum_{i=1}^{3} m_{i}\left(C_{i}\right) \omega_{i}(\varepsilon)=m_{1}\left(C_{1}\right) \omega_{1}(\varepsilon)+m_{2}\left(C_{2}\right) \omega_{2}(\varepsilon)+m_{3}\left(C_{3}\right) \omega_{3}(\varepsilon)=m_{1} \omega_{1}(\varepsilon)+m_{2} \omega_{2}(\varepsilon)+m_{3} \omega_{3}(\varepsilon) ;
$$

By Eq. (23),

$$
\omega_{1}(\varepsilon)=\frac{d_{2} d_{3}}{d_{1} d_{2}+d_{1} d_{3}+d_{2} d_{3}} ; \quad \omega_{2}(\varepsilon)=\frac{d_{1} d_{3}}{d_{1} d_{2}+d_{1} d_{3}+d_{2} d_{3}} ; \quad \omega_{3}(\varepsilon)=\frac{d_{1} d_{2}}{d_{1} d_{2}+d_{1} d_{3}+d_{2} d_{3}},
$$

and $\omega_{1}(\varepsilon)+\omega_{2}(\varepsilon)+\omega_{3}(\varepsilon)=1$. 
After associating primary materials to the contours accordingly, the layer is discretised into a set of small sub-regions; the property value of material composition of each sub-region is effectively calculated by Eqs. (22) and (23). Figure 21c shows the resulting layer with material composition variations along both radial and circumferential directions, while Figure 21d is the resulting FGM turbine blade with $3 \mathrm{D}$ change of material composition along the Z-plane and in radial and circumferential directions across the X-Y plane.

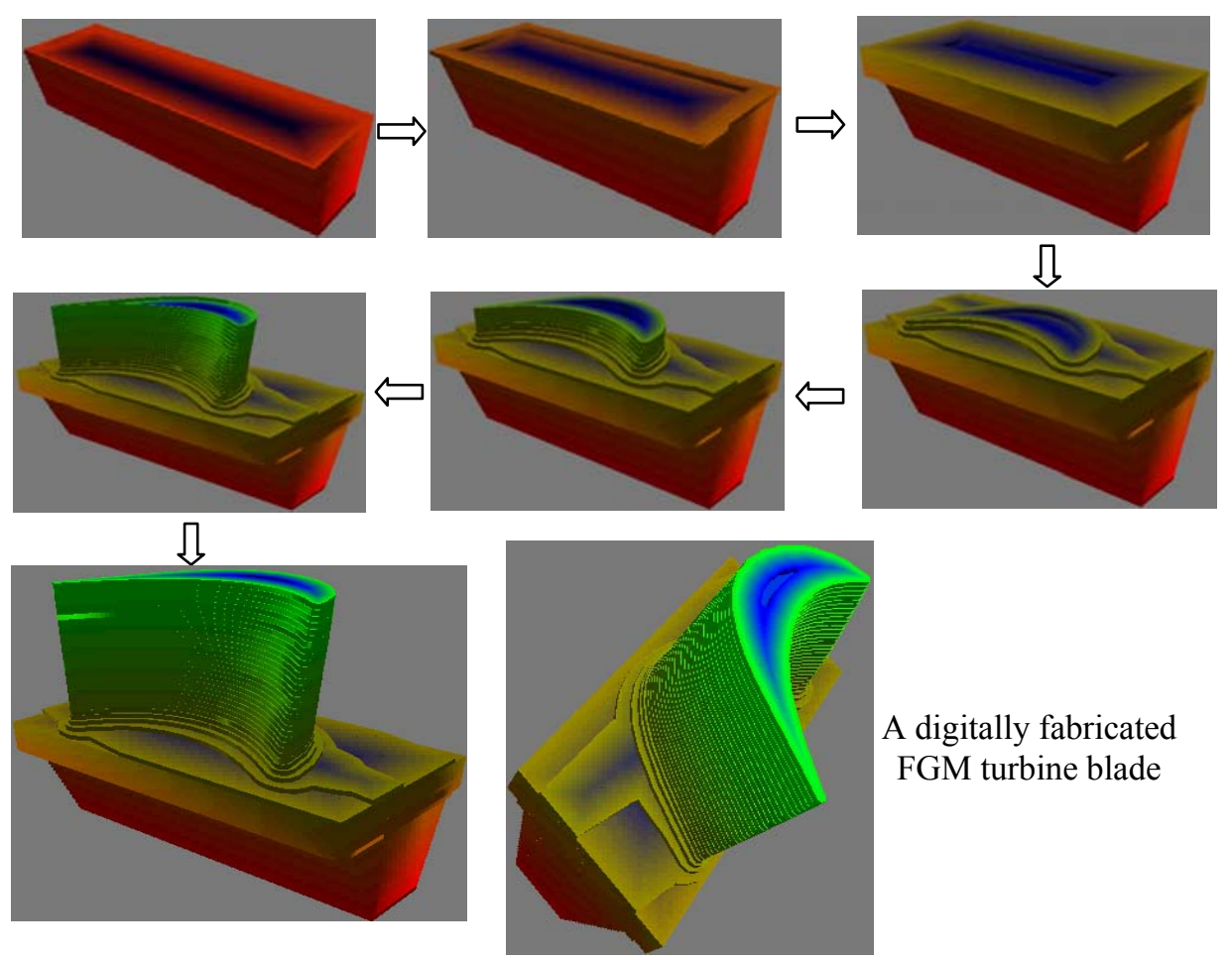

Figure 20. Digital fabrication of a turbine blade with linearly material composition changes along the Z-plane and in the $X-Y$ plane

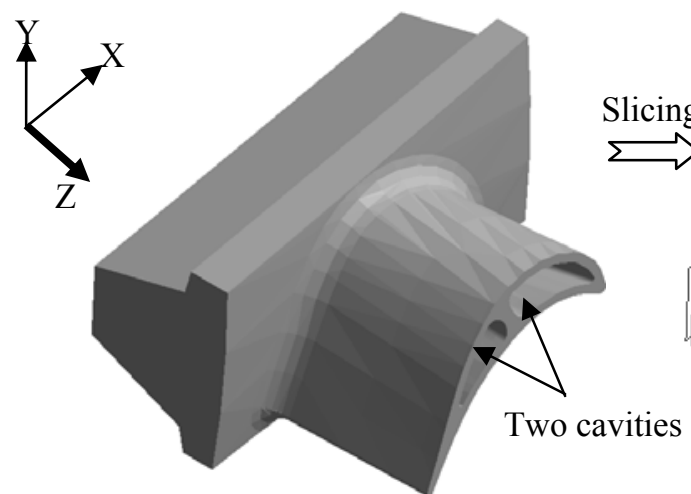

A homogeneous STL turbine blade with two cavities

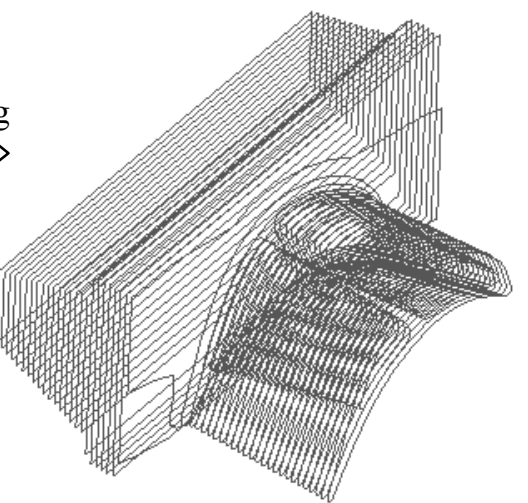

A homogeneous contour-based model

Figure 21a. A turbine blade with two cavities 


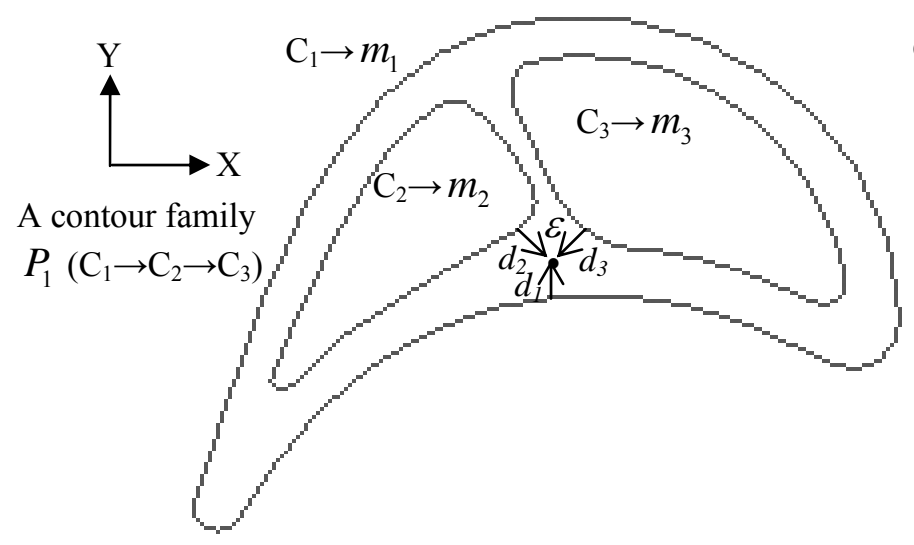

Figure 21b. A selected feature layer with three contours for assigning primary materials

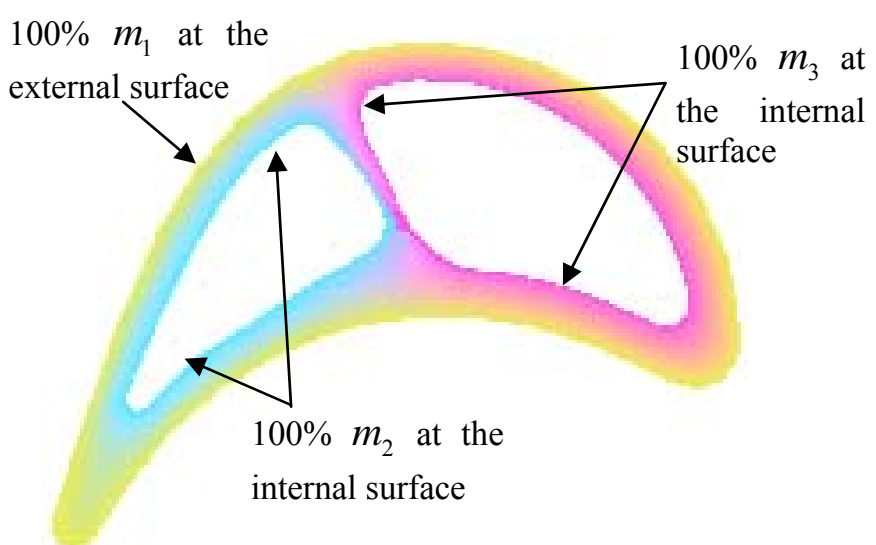

Figure 21c. Gradual change of material composition along both radial and circumferential directions in the $\mathrm{X}-\mathrm{Y}$ plane

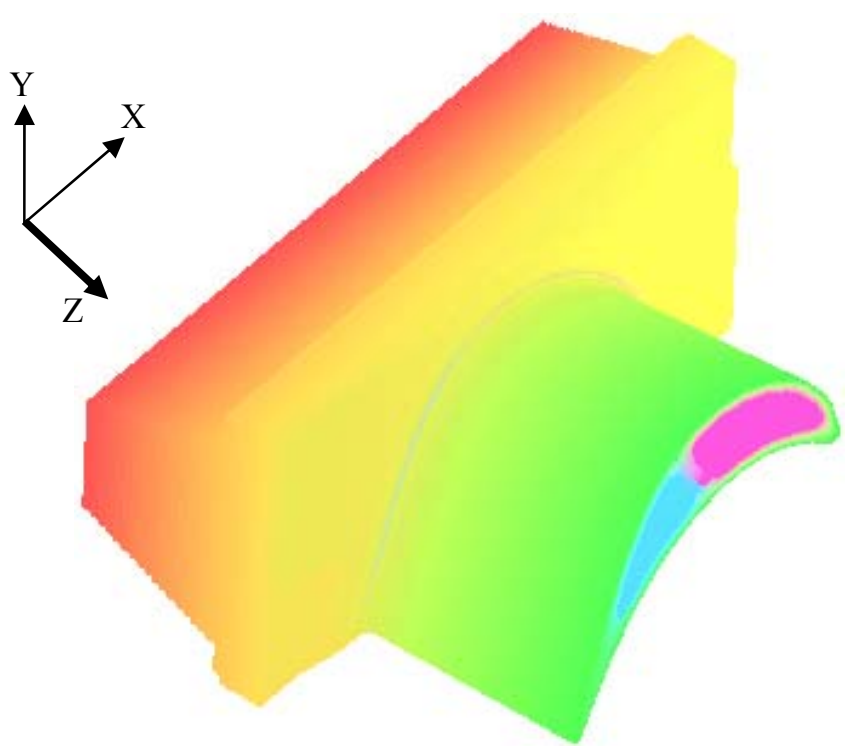

Figure 21d. An FGM turbine blade with 3D material composition variations

\subsection{Limitations of the proposed approach}

The case study above shows that the proposed approach, integrated with the FGMVP system, is a practical tool for representation, process planning, and subsequent fabrication of FGM objects. However, as an initial implementation, the proposed approach suffers some limitations. Indeed, it would be necessary to improve its robustness and capability to process more complex FGM objects. Firstly, the two discretisation methods for offsetting slice contours should be enhanced to process more complex, irregular concave contours. It would be useful to incorporate a more adaptive contour-offsetting algorithm for this purpose. Secondly, some intelligence should be incorporated such that when there is more than one contour in a slice in the 
$\mathrm{X}-\mathrm{Y}$ plane, it can automatically connect the appropriate corresponding contours between adjacent slices for modelling material composition variation along the Zplane. Therefore, it would be desirable to establish explicit inter-slice topological hierarchy information of slice contours for more convenient processing of complex FGM objects.

\subsection{Conclusions}

This paper presents a topological hierarchy-based approach to layered manufacturing of FGM objects. The approach has been implemented to form a virtual prototyping (FGMVP) system for design, process planning, and subsequent fabrication of FGM objects. The approach represents an FGM object with material control functions specified across slice contours. Based on the topological hierarchy information of slice contours, material control functions can be conveniently associated with contour families of some representative layers across the X-Y plane and along the Z-plane. The material composition at any point can be easily evaluated with the control functions. Besides, two discretisation methods are developed to divide 2D slices into uniform and non-uniform sub-regions of constant material composition for visualisation of MMLM processes, and subsequent fabrication of FGM objects. The discretisation resolution can be flexibly varied to suit visualisation and fabrication requirements. In comparison with pixel- or voxel-based representation schemes, the proposed approach is versatile, efficient and requires little memory to process large and complex objects of both FGM and discrete materials. More importantly, it facilitates planning of multi-material toolpaths for process control of MMLM machines. Therefore, the FGMVP system is a practical and effective tool for design visualisation and subsequent fabrication of FGM objects, though further effort is needed to improve its robustness and processing capacity.

\section{Acknowledgements}

The authors would like to acknowledge the Research Grant Council of the Hong Kong SAR Government and the CRCG of the University of Hong Kong for their financial support for this project.

\section{References}

1 V. Kumar, Solid modeling and algorithms for heterogeneous objects, Ph.D. Thesis, The University of Michigan, USA, (1999). 
2 K.H. Shin, D. Dutta, Constructive representation of heterogeneous objects, Journal of Computing and Information Science in Engineering 1(3) (2001) 205217.

3 J. Jepson, J.J. Beaman, D.L. Bourell, K.L. Wood, SLS processing of functionally gradient materials, in: D.L. Bourell, et al. (Eds.), Solid Freeform Fabrication Symposium, Austin, Texas. The University of Texas, 1(997), pp. 1-8.

4 K. Lappo, B. Jackson, K. Wood, D.L. Bourell, J.J. Beaman, Discrete multiple material selective laser sintering $\left(\mathrm{M}^{2} \mathrm{SLS}\right)$ : experimental study of part processing, in: D.L. Bourell, et al. (Eds.), Solid Freeform Fabrication Symposium, Austin, Texas, The University of Texas, (2003), pp. 109-119.

5 R. Merz, F.B. Prinz, K. Ramaswami, M. Terk, L.E. Weiss, Shape deposition manufacturing, in: D.L. Bourell, et al. (Eds.), Solid Freeform Fabrication Symposium, Austin, Texas, The University of Texas, (1994), pp. 1-8.

6 J.R. Fessler, A.H. Nickel, G.R. Link, F.B. Prinz, P. Fussell, Functional gradient metallic prototypes through shape deposition manufacturing, in: D.L. Bourell, et al. (Eds.), Solid Freeform Fabrication Symposium, Austin, Texas, The University of Texas, (1997), pp. 521-528.

7 M.A. Jafari, W. Han, F. Mohammadi, A. Safari, S.C. Danforth, N. Langrana, A novel system for fused deposition of advanced multiple ceramics, Rapid Prototyping Journal 6(3) (2000) 161-175.

8 T.R. Jackson, H. Liu, N.M. Patrikalakis, E.M. Sachs, M.J. Cima, Modeling and designing functionally graded material components for fabrication with local composition control, Materials \& Design 20(2-3) (1999) 63-75.

9 W.J. Cho, E.M. Sachs, N.M. Patrikalakis, D.E. Troxel, A dithering algorithm for local composition control with three-dimensional printing, Computer-Aided Design 35(9) (2003) 851-867.

10 J.W. Wang, L.L. Shaw, Fabrication of functionally graded materials via inkjet color printing, Journal of the American Ceramic Society 89(10) (2006) 3285-3289.

11 V. Kumar, D. Dutta, An approach to modeling \& representation of heterogeneous objects, Transactions of the ASME, Journal of Mechanical Design 120(3-4) (1998) 659-667.

12 W.K. Chiu, S.T. Tan, Multiple material objects: from CAD representation to data format for rapid prototyping, Computer-Aided Design 32(12) (2000) 707-717.

13 T.R. Jackson, Analysis of functionally graded material object representation methods, Ph.D. Thesis, Massachusetts Institute of Technology, USA, (2000).

14 Y.K. Siu, S.T. Tan, 'Source-based' heterogeneous solid modelling, ComputerAided Design 34(1) (2002) 41-55.

15 M.Y. Zhou, J.T. Xi, J.Q. Yan, Modeling and processing of functionally graded materials for rapid prototyping, Journal of Materials Processing Technology 146(1-3) (2004) 396-402.

16 X.Y. Kou, S.T. Tan, A hierarchical representation for heterogeneous object modelling, Computer-Aided Design 37(3) (2005) 307-319.

17 Y.K. Siu, S.T. Tan, Modeling the material grading and structures of heterogeneous objects for layered manufacturing, Computer-Aided Design 34(10) (2002) 705-716.

18 K.H. Shin, D. Dutta, Process-planning for layered manufacturing of heterogeneous objects using direct metal deposition, Transactions of the ASME, Journal of Computing and Information Science in Engineering 2(4) (2002) 330344. 
19 X.Y. Kou, Computer-Aided design of heterogeneous objects, Ph.D. Thesis, The University of Hong Kong, Hong Kong, (2005).

20 Pixel, http://en.wikipedia.org/wiki/Pixel, 2008.

21 S.H. Choi, K.T. Kwok, A topological hierarchy-sorting algorithm for slice contours, Rapid Prototyping Journal 10(2) (2004) 98-113.

22 A. Biswas, V. Shapiro, I. Tsukanov, Heterogeneous material modeling with distance fields, Computer Aided Geometric Design 21(3) (2004) 215-242.

23 S.H. Choi, H.H. Cheung, A multi-material virtual prototyping system, ComputerAided Design 37(1) (2005) 123-136.

24 S.H. Choi, H.H. Cheung, A topological hierarchy-based approach to toolpath planning for multi-material layered manufacturing, Computer-Aided Design 38(2) (2006) 143-156.

25 S.H. Choi, H.H. Cheung, A CAVE-based multi-material virtual prototyping system, Computer-Aided Design and Applications 3(5) (2006) 557-566.

26 S.H. Choi, H.H. Cheung, Multi-material virtual prototyping for product development and biomedical engineering, Computers in Industry 53(5) (2007) 438-452.

27 X.P. Qian, D. Dutta, Design of heterogeneous turbine blade, Computer-Aided Design 35(3) (2003) 319-329.

28 S. Das , T.P. Fuesting, G. Danyo, L.E. Brown, J.J. Beaman, D.L. Bourell, Direct laser fabrication of superalloy cermet abrasice turbine blade tips, Materials \& Design 21(2) (2000) 63-73. 